\title{
Effect of softening function on the cohesive crack fracture parameters of concrete CT specimen
}

\author{
SHAILENDRA KUMAR and S V BARAI* \\ Department of Civil Engineering, Indian Institute of Technology, \\ Kharagpur 721302 \\ e-mail: (shailendra, skbarai)@ civil.iitkgp.ernet.in
}

MS received 14 November 2008; revised 24 June 2009

\begin{abstract}
The paper presents numerical study on the fracture parameters of concrete compact tension test specimens of different sizes using cohesive crack model. As softening function is the main ingredient of the cohesive crack model, seven numbers of widely used softening functions are incorporated in the model based on enhanced algorithm. It is found that the difference between the highest and the lowest peak loads obtained using various softening functions (except linear) is less than about $9 \%$. The peak load predicted by the linear softening curve is about $16 \%$ larger than that of mean peak load predicted by other softening functions. The cohesive crack model with linear softening yields the fracture process zones lower by approximately $30-50 \%$ than those obtained by using the other softening relations for specimen size range $200-600 \mathrm{~mm}$. The numerical results are further compared with a reference test result available in the literature. It is observed that some of softening relations (except linear) predict the experimentally obtained peak load up to $6 \%$ of accuracy whereas the linear softening curve overestimates it by about $30 \%$. The numerically gained softening branch of load-displacement curves compare well with the experimental observation.
\end{abstract}

Keywords. Fracture process zone; cohesive crack method; softening function; compact tension test; size effect.

\section{Introduction}

In early 1960s application of classical linear elastic fracture mechanics (LEFM) (Kaplan 1961) was initially attempted to measure fracture toughness of plain concrete notched beams of different sizes tested in three and four point bend configurations. This pioneering work subsequently attracted attention of many researchers to determine the critical stress intensity factor $K_{I C}$ of cementitious materials similar to metals. Later it was concluded that these materials did not possess a consistent value of the critical stress intensity factor (Kesler et al 1972; Walsh 1972; Walsh 1976) with respect to structural size and therefore the concept of

*For correspondence 
LEFM was inadequate to describe the fracture behaviour of concrete. It is almost settled that LEFM is only applicable to large-mass concrete structures and not applicable to medium and small-scale concrete structures. The inapplicability of LEFM is attributed to the presence of large fracture process zone (FPZ) of variable size ahead of the crack tip and the cohesive stress transferred within the FPZ of the quasibrittle materials like concrete.

The complex nonlinear phenomena that takes place in the fracture process zone can be idealized and approximated using nonlinear fracture approaches to predict the localized real physical behaviour in the vicinity of a crack and at the crack tip. The cohesive crack model is one of such simplified nonlinear fracture models that can simulate satisfactorily the behaviour of concrete fracture. Inspired by the early stage of development of the fracture models (Barenblatt 1959; Dugdale 1960), Hillerborg and co-workers (Hillerborg et al 1976) initially applied cohesive crack method (or fictitious crack model) as a suitable nonlinear model for mode I fracture to simulate the softening damage of concrete structures. In the pioneering work they showed that analysis of crack formation and propagation as well as failure analysis could be done with cohesive crack model even if coarse finite element was used thereby eliminating the mesh sensitivity. Ever since, a number of nonlinear fracture models: crack band model (CBM) (Bažant \& Oh 1983), two parameter fracture model (TPFM) (Jenq \& Shah 1985), size effect model (SEM) (Bažant et al 1986), effective crack model (ECM) (Nallathambi \& Karihaloo 1986), $K_{R}$-curve based on cohesive force (Xu \& Reinhardt 1999a), double-K fracture model (DKFM) (Xu \& Reinhardt 1999a; 1999b) and double-G fracture model (DGFM) (Xu \& Zhang 2008) have been proposed and used to predict fracture behaviour of concrete.

The nonlinear fracture models are based on two basic approaches: first using finite element method (FEM) or boundary element method and second using modified LEFM concept. The cohesive crack model and crack band model fall under the former category while two parameter fracture model, size effect model, effective crack model, $K_{R}$-curve based on cohesive force, double-K fracture model and double-G fracture model belong to latter group. The simplified assumptions and the ease with which finite element method or boundary element method is applied in formulation of cohesive crack model are the main reasons of its popularity. In contrast to nonlinear models based on LEFM, cohesive crack model can satisfactorily capture the behaviour of an uncracked structure from crack initiation to the failure. The essential ingredient of the cohesive crack model is to use a proper shape of tension-softening relation (softening function) to characterize the fracture behaviour of cementitious materials. Figure 1 represents the general characteristics of a softening function of concrete; in particular, it shows the variation of cohesive stress with respect to the crack opening displacement in the process zone. Material tensile strength $f_{t}$ and the critical crack width $w_{c}$ are the two important characteristic values of the softening function and the area under the curve is defined as the total fracture energy $G_{F}$ of the material. The fracture energy $G_{F}$ is determined from a load-displacement curve obtained from a stable three-point bend test (TPBT) in accordance with RILEM guidelines (RILEM Technical Committee 50-FMC 1985). The fracture properties can also be determined using compact tension (CT) test (Wittmann et al 1988) specimen and the wedge splitting test (WST) (Brühwiler \& Wittmann 1990). Each method of measuring fracture properties however is associated with some advantages and limitations. For large size structures, the self-weight of the specimen possibly negates the use of the TPBT for fracture testing not only due to handling problems of testing specimens, but also it requires special care in fracture analysis. The following advantages were suggested (Wittmann et al 1988) in determining the specific fracture energy $G_{F}$ of concrete using compact tension (CT) test and bilinear softening function as compared with the RILEM recommendation. 


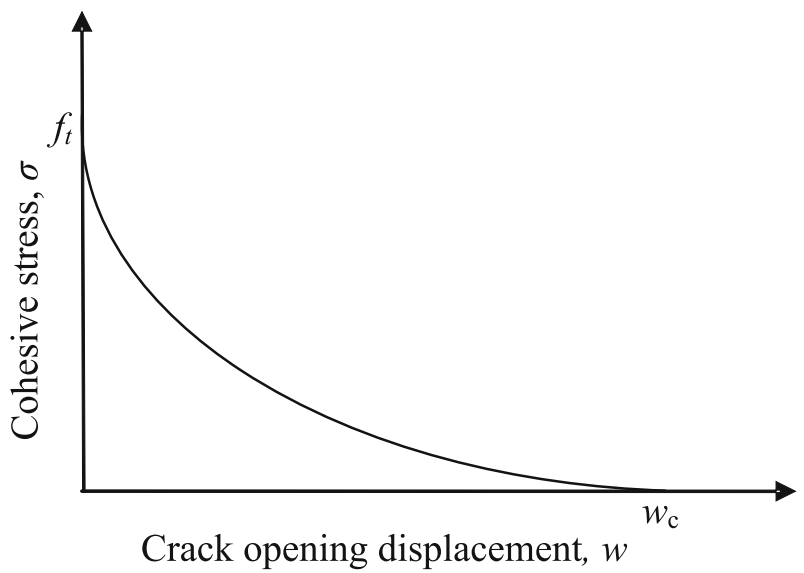

Figure 1. Representation of softening function of concrete.

- Any load-displacement curve along the notch can be used.

- The end point load-displacement curve is not needed.

- No correction due to self-weight is required.

The ASTM standard E399-06 (2006) has specified the general proportion and standard configuration of CT specimen which is shown in figure 2. According to the ASTM standard E-399-06 (2006), the dimensions of standard CT specimen should satisfy: $D_{1}=1 \cdot 25 D, H=$ $0.6 D, H_{1}=0.275 D$ and the specimen thickness $B=0.5 D$. It is also known that the shape of softening function affects the fracture behaviour of concrete. Though studies using cohesive crack model for predicting fracture behaviour of concrete are found in literature, a systematic study using seven numbers of important softening functions is carried out in present paper. Cohesive crack model based on enhanced algorithm for mode I fracture of concrete CT

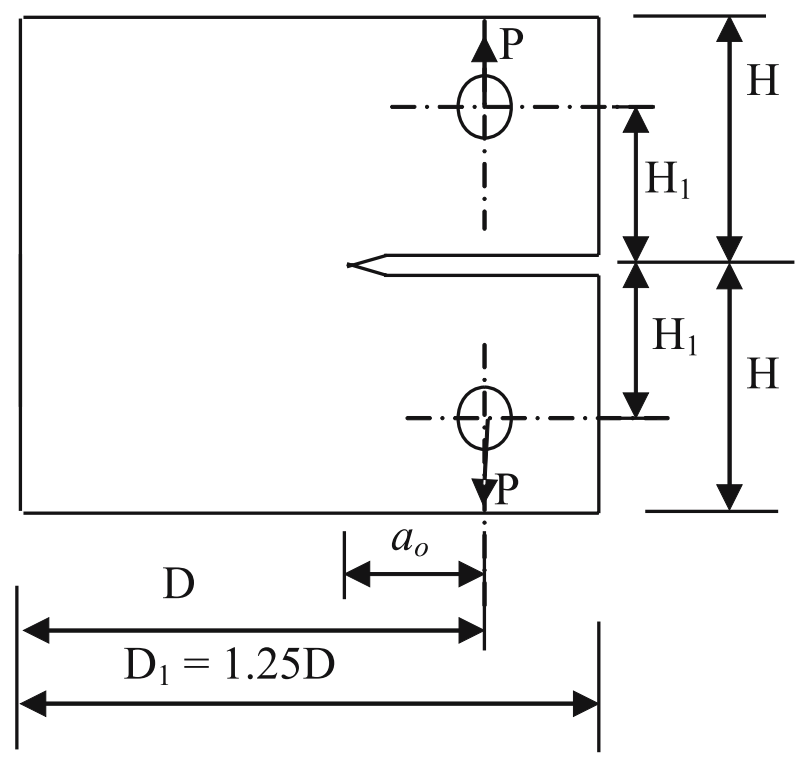

Figure 2. Standard geometry of compact tension (CT) specimen. 


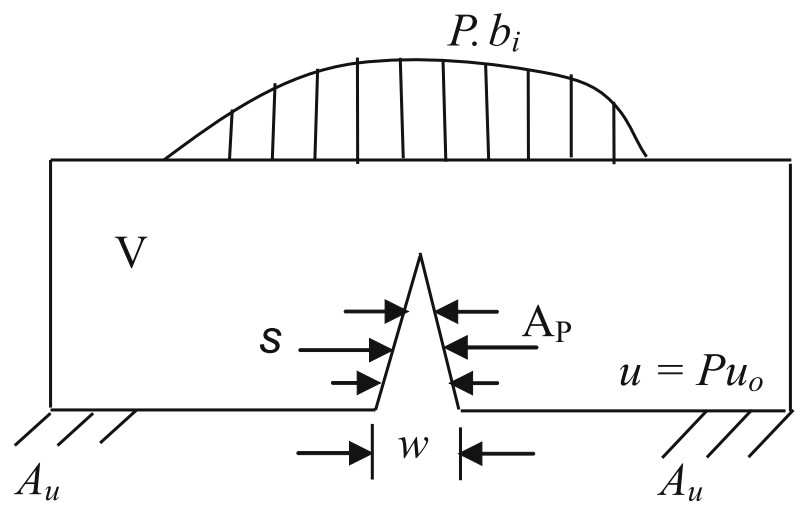

Figure 3. Representation of potential energy definition of a cracked body.

specimen is developed. The nonlinear simultaneous equations for cohesive crack model are written with the help of procedures available in literature (Petersson 1981; Carpenteri 1989; Carpenteri \& Colombo 1989) and the solution of the same are obtained using enhanced influence method (Planas \& Elices 1991). Then, a comparative study of global response and localized fracture behaviour in the process zone and at crack-tip influenced by different shapes of softening functions for the compact tension specimen of size range $100 \leq D \leq 600 \mathrm{~mm}$ is presented. The study reports the effect of different softening curves on the prediction of peak load, behaviour showing size-scale transition towards linear elastic fracture mechanics, sizeeffect study, development of fracture process zone and corresponding load bearing capacity, and fictitious crack length, crack tip opening displacement and local stress variation at initial notch tip corresponding to peak load. At the end, numerically simulated load-displacement curves using different softening functions are compared with those obtained from a standard test result available in the literature.

\section{Cohesive crack model (CCM)}

\subsection{Formulation based on energy principle}

Assuming that no unloading occurs in the fracture process zone-under condition of proportional and monotonic loading in mode I crack propagation, the process-zone boundary can be defined by only one variable i.e. the crack length $a$. The potential energy $\Pi$ of the system (figure 3) is written as (Li \& Liang 1994):

$$
\Pi\left(u_{i}, P, a\right)=\int_{V} W\left(\varepsilon_{i j}\right) d V-\int_{V} F_{i} u_{i} d V-P \int_{A_{T}} b_{i} u_{i} d A+\int_{A_{P}} \phi(w) d A .
$$

Where, $W=$ the strain energy density function defined in the body, $\varepsilon_{i j}=$ strain field, $F_{i}=$ the body force, $u_{i}=$ an admissible displacement field for the system, $b_{i}=$ boundary force distribution defined in the part of boundary $A_{T}$. The potential $\phi$ can be defined for any given cohesive law $\sigma=f(w)$ as:

$$
\phi(w)=\int_{0}^{w} f(v) d v
$$


The positive sign of the surface potential is due to the fact that the positive direction of cohesive forces is in the opposite direction to that of the crack opening displacement. The displacement equilibrium of the system is obtained by equating to zero the first-order variation of the potential $\Pi$ with respect to displacement.

$0=\Pi_{u} \cdot \delta u_{i}=\int_{V} \sigma_{i j} \delta \varepsilon_{i j} d V-\int_{V} F_{i} \delta u_{i} d V-P \int_{A_{T}} b_{i} \delta u_{i} d A+\int_{A_{P}} f(w) \delta w d A$.

All possible equilibrium states of the system cannot be determined using (3). Among all the displacement equilibrium states that satisfy (3) for different combinations of given $P$ and $a$, there is one combination which satisfies the following crack equilibrium equation:

$$
\begin{aligned}
0= & \Pi_{a}=\frac{\partial \Pi}{\partial a}=\frac{\partial}{\partial a}\left(\int_{V} W\left(\varepsilon_{i j}\right) d V-\int_{V} F_{i} u_{i} d V-P \int_{A_{T}} b_{i} u_{i} d A\right) \\
& +\frac{\partial}{\partial a} \int_{A_{P}} \phi(w) d A, \quad \forall \delta a>0 .
\end{aligned}
$$

For linear elastic body and constant body forces, the terms in the parenthesis on the righthand side of (4) can be recognized as the energy release rate, whereas the last term is the energy needed to create a unit area of process zone, which is deformation dependent and not as constant material property. Hence, (4) is equivalently written in terms of stress intensity factors as:

$$
\left(P k_{P}+K_{\sigma}\right)^{2}=0 \text { or } P k_{P}+K_{\sigma}=0 .
$$

Where, $k_{P}$ is the stress intensity factor at the process zone tip due to unit applied load, $P=1$ and $K_{\sigma}$ is the stress intensity factor at the process zone tip due to cohesive stress in the process zone. The basic features of cohesive crack models are represented using (5) that is total stress intensity factor at the crack tip due to external loading, constant body force, boundary force, if any and cohesive stress distribution in the process zone is zero. This statement eliminates the stress singularity at the crack tip of the structure and a finite stress acts near the crack tip.

\subsection{Basic assumptions}

The following assumptions are considered in the development of cohesive crack model (Petersson 1981; Carpenteri 1989; Carpenteri \& Colombo 1989).

(i) The bulk of material behaves in a linear elastic and isotropic manner.

(ii) The cohesive process zone begins to develop when the maximum principal stress becomes equal to the tensile strength.

(iii) The material is in partial damaged condition and is still able to transfer the stress known as cohesive stress $\sigma$, after formation of cohesive fracture zone. The cohesive stress depends on the crack opening displacement $w$. For mode I opening, $\sigma$ acts normal to the crack faces as shown in figure 4 and is related to $w$ as below:

$$
\sigma=f(w)
$$

Also from definition of softening function of concrete, we have

$$
f_{t}=f(0)
$$




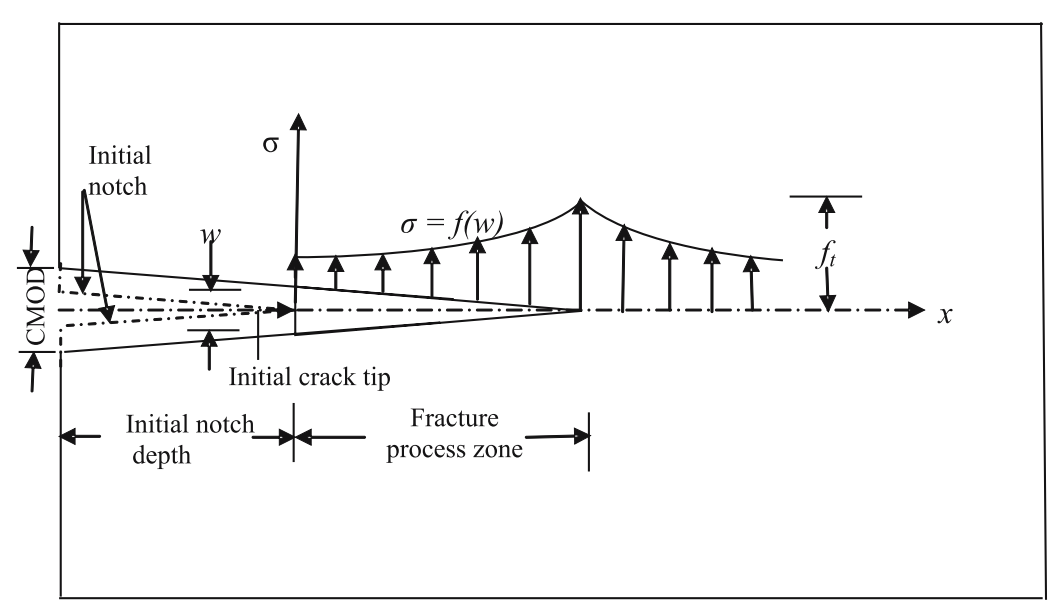

Figure 4. Stress distribution and cohesive crack growth in mode-I opening for concrete.

and total fracture energy $G_{F}$ is mathematically written as

$$
G_{F}=\int_{0}^{w_{c}} f(w) d w .
$$

The structural behaviour can be analysed using the parameter called characteristic length $l_{c h}$.

$$
l_{c h}=\frac{E G_{F}}{f_{t}^{2}},
$$

where, $E$ is the modulus of elasticity of concrete. The characteristic length is an inverse measure of the brittleness of the material.

\subsection{Finite element discretization}

Two material lengths (i) the fracture process zone and (ii) the width of the FPZ have always been a matter of research in the fracture modelling of concrete. Experimental results (Petersson 1981) show that the size of FPZ is significant and comparable but its width is normally small (in the order of maximum aggregate size) with respect to the characteristic dimension of a structure. Depending on the method of analysis, these material lengths are considered or ignored during numerical simulation. In the crack band model (Bažant \& Oh 1983), the width of FPZ is one of material parameters and taken to be three times the maximum aggregate size. The fictitious crack model or cohesive crack model assumes the FPZ collapsed into a line or a surface in two or three-dimensional analysis, respectively. Cohesive crack modelling using FEM can be done in a natural way with relatively few degrees of freedom employing interface elements with many advantages (Ingraffea et al 1984; Bocca et al 1991; Gerstle \& Xie 1992; Xie \& Gerstle 1995; Prasad \& Krishnamoorthy 2002). The interface elements takes into account the normal compression and shear tractions between the crack faces of an alreadyformed crack and the cohesive tractions in the process zone. However, continuous remeshing or topological variation during crack propagation is a burdensome need in case of numerical modelling of mixed mode problems. To avoid this difficulty, Alfaiate et al (1997) developed a 


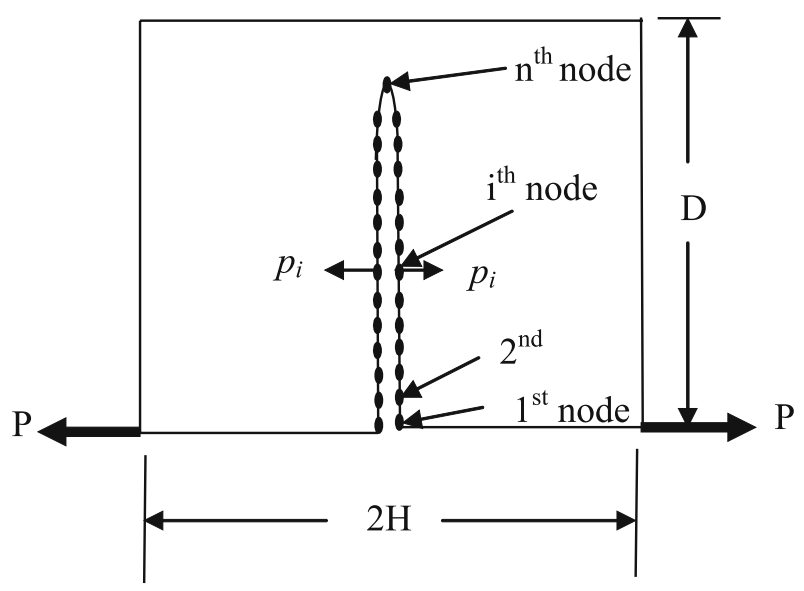

Figure 5. Finite element pair of nodes along fracture line.

method for non-prescribed crack propagation in concrete, in which, the interface elements of zero thickness were introduced without remeshing and the properties of the crack path were projected into the fixed element boundaries. When a single macroscopic crack opens in a fixed direction under a pure mode I loading, the cohesive crack can be modelled easily by employing procedures proposed by Petersson (1981) and further modified by Carpinteri (1989) and Planas \& Elices (1991). In this method the number of fracture nodes along the potential crack line is kept to be fixed without introducing the interface elements in the FEM. Only a standard linear finite element analysis is needed to compute the required influence coefficients. Further, a system of nonlinear simultaneous equation due to introduction of softening function is developed and solved using a standard method for the study of crack propagation. It may be pointed out that the fracture parameters remain the same and independent of the methods whether interface elements are used or not in the analysis. The basic aim of the present contribution is to study the influence of different softening function on the fracture behaviour of mode I problem in which the path of crack propagation in cohesive crack modelling is known a priori. With this limitation, the nonlinear behaviour of CT specimen is simulated without introducing interface elements in the finite element analysis.

Due to symmetry of CT specimen, the potential fracture line is shown in figure 5. The crack opening nodes are taken from 1 at the bottom to $n$ at the top along the potential fracture line. The crack opening displacements along the fracture line due to external force of the structure are grouped in the column matrix $w$ and corresponding nodal forces into a column matrix $F$. The other remaining nodal displacement and nodal forces for rest of the structure are grouped into column matrices $w_{R}$ and $F_{R}$. Then partitioned form of associated finite element equation can be written as:

$$
\left\{\begin{array}{l}
F \\
F_{R}
\end{array}\right\}=-\left[\begin{array}{ll}
K_{C C} & K_{C R} \\
K_{R C} & K_{R R}
\end{array}\right]\left\{\begin{array}{l}
w \\
w_{R}
\end{array}\right\} .
$$

Considering that no other forces are acting on the rest of the specimen, $\left[F_{R}\right]=0$, and eliminating out all the displacement components of $w_{R}$, the matrix equilibrium equation is obtained as:

$$
\{F\}=\left[K_{F}\right]\{w\}
$$


in which,

$$
\left[K_{F}\right]=\left[K_{C C}\right]-\left[K_{C R}\right]\left[K_{R R}\right]^{-1}\left[K_{R C}\right] .
$$

The crack opening for $n$ nodes along the fracture zone is further expressed in the following form for convenience.

$$
\{w\}=[K]\{p\}+\{C\} P,
$$

where, $\{w\}=$ a vector of crack opening displacement at node $i$.

$[K]=$ a symmetric matrix and the value of $K_{i j}$ is the crack opening displacement at node $i$ by a unit opening nodal force applied at node $j$.

$\{p\}=$ a vector of nodal forces.

$\{C\}=$ a vector of crack opening displacement at node $i$ when $P=$ unit value.

In the present study, the enhanced influence method (Planas \& Elices 1991) is used to solve (13). Assuming that the total number of fracture nodes are $n$ and initial crack tip lies at $k^{\text {th }}$ node, then according to this method, (13) may be partitioned between the notches from nodes, $i=1,2,3, \ldots,(k-1)$ in the notch portion and from $i=k,(k+1),(k+2), \ldots, n$ in the ligament portion. Hence, the partitioned (13) may be written as:

$$
\left\{\begin{array}{l}
w_{N} \\
w_{L}
\end{array}\right\}=\left[\begin{array}{c}
K_{N N} K_{N L} \\
K_{L N} K_{L L}
\end{array}\right]\left\{\begin{array}{l}
p_{N} \\
p_{L}
\end{array}\right\}+\left\{\begin{array}{l}
C_{N} \\
C_{L}
\end{array}\right\} P .
$$

The subscripts $N$ and $L$ denote notch portion for $i=1,2, \ldots,(\mathrm{k}-1)$ and ligament zone for $i=k,(k+1),(k+2), \ldots, n$. Since width of crack opening in ligament zone is zero and initial notch portion is traction free zone, it may be written as:

$$
\left.\begin{array}{l}
\left\{p_{N}\right\}=\{0\} \text { for } i=1,2,3, \ldots,(k-1) \\
\left\{w_{L}\right\}=\{0\} \text { for } i=k,(k+1),(k+2), \ldots, n
\end{array}\right\}
$$

The (14) and (15) yield

$$
\left\{p_{L}\right\}=\left[M_{L L}\right]\left\{w_{L}\right\}-\left[M_{L L}\right]\left\{C_{L}\right\} P,
$$

where, $M_{L L}=K_{L L}^{-1}$ and $\left\{T_{L}\right\}=\left[M_{L L}\right]\left\{C_{L}\right\}$

$$
\left\{p_{L}\right\}=\left[M_{L L}\right]\left\{w_{L}\right\}-\left\{T_{L}\right\} P .
$$

Further, the ligament portion may be partitioned between cohesive zone and uncracked (or undamaged) zone along the fracture line. Let cohesive zone forms between the nodes $j=$ $k,(k+1), \ldots, l$ and undamaged part is $j=(l+1) \cdot(l+2), \ldots, n$. Hence after further partitioning of (17), it may be written as:

$$
\left\{\begin{array}{l}
p_{L C} \\
p_{L U}
\end{array}\right\}=\left[\begin{array}{ll}
M_{L L C C} & M_{L L C U} \\
M_{L L U C} & M_{L L U U}
\end{array}\right]\left\{\begin{array}{l}
w_{L C} \\
w_{L U}
\end{array}\right\}-\left\{\begin{array}{l}
T_{L C} \\
T_{L U}
\end{array}\right\} P .
$$

Where, subscripts $C$ and $U$ denote cohesive zone for $j=k,(k+1), \ldots, l$ and undamaged zone for $j=(l+1) \cdot(l+2), \ldots, n$. Since width of crack opening displacements in undamaged zone and also at the last node of cohesive zone are zero, mathematically it is expressed as:

$$
\left.\begin{array}{l}
\left\{w_{L U}\right\}=\{0\} \\
\left\{w_{L C}\right\}_{j=l}=0
\end{array}\right\} .
$$


The (18) and (19) yield

$$
\left\{p_{L C}\right\}=\left[M_{L L C C}\right]\left\{w_{L C}\right\}-\left\{T_{L C}\right\} P .
$$

For $j=l^{\text {th }}$ node, the applied load $P$ may be expressed as:

$$
P=\frac{\sum_{j=k}^{l-1}\left\{M_{L L C C}\right\}_{l, j}\left\{w_{L C}\right\}_{j}-\left\{p_{L C}\right\}_{j=l}}{\left\{T_{L C}\right\}_{j=l}} .
$$

For the nodes, $j=k,(k+1),(k+2), \ldots,(l-1)$ and using (20) and (21), it can be formed as many number of nonlinear simultaneous equations as the cracks are opened in the cohesive zone. The functional form of the nonlinear simultaneous equations may be expressed as:

$$
\{Z\}=\left[M_{L L C C}\right]\left\{w_{L C}\right\}-\left\{p_{L C}\right\}-\left\{T_{L C}\right\} \frac{\sum_{j=k}^{l-1}\left\{M_{L L C C}\right\}_{l, j}\left\{w_{L C}\right\}_{j}-\left\{p_{L C}\right\}_{j=l}}{\left\{T_{L C}\right\}_{j=l}} .
$$

If proper sign convention is used, it can be written as, $\left\{p_{L C}\right\}=-F_{u c} f\left(w_{L C}\right)$, where for regular discretization of uniform mesh size $h, F_{u c}=B h f_{t}$ for all values of $i$ except, $i=k$ and $i=n$; and for $i=k$ and $i=n, F_{u c}=\frac{B h f_{t}}{2}$. The solution of (22) is obtained using Newton-Raphson method. The function $Z$ and its Jacobian matrix for unknown crack opening displacements using different softening functions are programmed in separate functions. Thus, this method becomes powerful and as many types of softening laws as possible can be incorporated in a program. After the solution of (22) for $\left\{w_{L C}\right\}$, the values of $P$ and $\left\{p_{L C}\right\}$ can be determined using (21) and (20) respectively. Going back to (19), (18), (17), (15), (14) and (13), it is possible to determine all unknown values of $\{w\}$ and $\{p\}$.

The values $[K]$ and $\{C\}$ of (13) are the known quantities and can be computed using standard linear elastic finite element method. Half of the specimen is discretized due to symmetry using four noded isoparametric quadrilateral elements as shown in figure 6 . For the sake of simplicity in element connectivity and nodal co-ordinate, the specimen is divided into three bands of length $0 \cdot 075 \mathrm{D}, 0 \cdot 225 \mathrm{D}$ and $0 \cdot 3 \mathrm{D}$ respectively along its longitudinal direction. The number of meshes in each band is taken to be 6,18 and 6 respectively. However, these numbers can be chosen arbitrarily to any value depending upon the memory capacity of the computer. The number of divisions along depth of the specimen is fixed as 80 . Three numbers of nodes from the top in horizontal direction and the top end in vertical direction are restrained against movement to allow the development of cohesive zone along potential fracture line. Then number of elements, number of degrees of freedom (d.o.f.) and number of fracture nodes along the potential fracture line become 2400,5022 and 78 respectively.

\subsection{Model implementation}

Firstly, the linear elastic finite element program is executed to obtain the influence coefficients such as $[K]$ and $\{C\}$. These coefficients are determined once and for all and used for cohesive crack propagation program. The set of nonlinear simultaneous equations for cohesive crack model are solved using enhanced influence method (Planas \& Elices 1991). Since the structure is linear elastic the tensile stress at $k^{\text {th }}$ node will exceed the tensile strength of concrete and this node is opened first. Thereafter, cohesive stress starts to act across the opened node pair according to a particular softening law. At this point the basic unknown quantities viz. $\{w\},\{p\}$ 


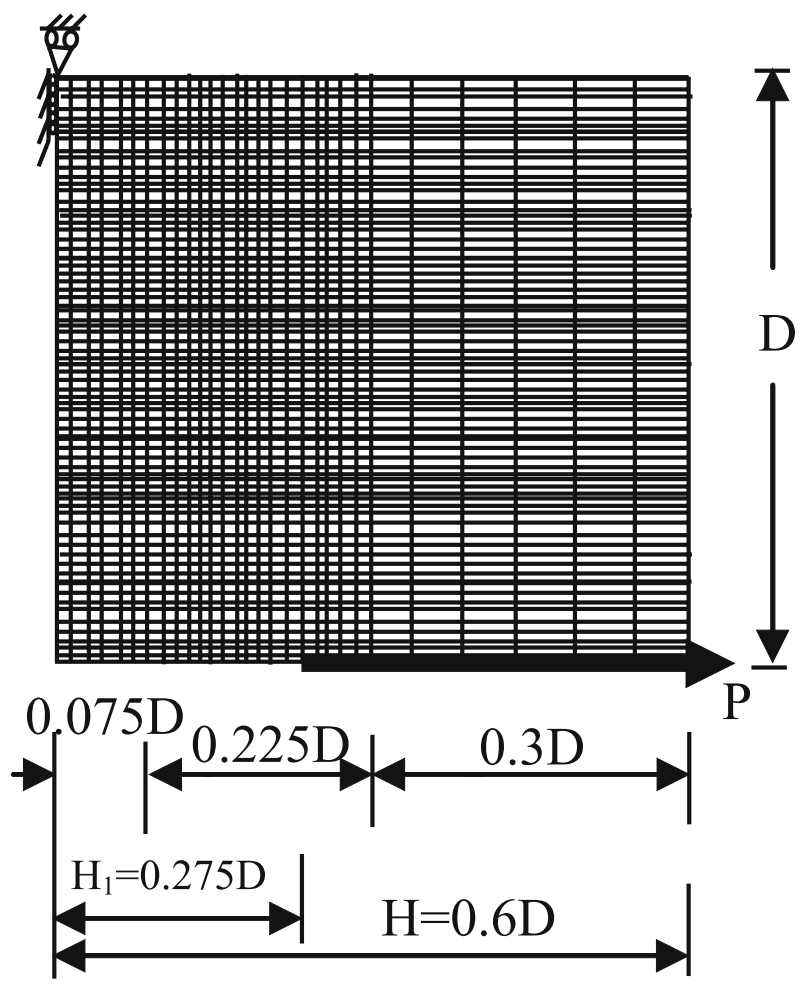

Figure 6. Finite element mesh for half of the CT specimen.

and $P$ are determined. In this step the first point of P-COD (load-load point crack opening displacement) curve is thus determined. In the second stage of calculation, the external load is increased to some particular value so that the tensile stress at node $(k+1)$ becomes greater than tensile strength of concrete. At this stage, $(k+1)^{\text {th }}$ nodes pair also get opened and cohesive stress starts to act at this node. Following similar process as mentioned above, the second point of P-COD curve is obtained. The number of basic unknowns is greatly reduced with enhanced algorithm in which assuming that number of nodes lies in cohesive zone are equal to $l$. Then the similar process is continued until the nodes pair $(l-1)$ in cohesive zone is opened and the program is terminated when $l=n$. The basic unknowns in every step are as many as the numbers of nodes opened in the cohesive zone. At each step of computation the value of crack opening displacement is checked and when it becomes greater than $w_{c}$ the corresponding value of nodal force goes down to zero.

\section{Softening function of concrete}

Some widely used softening functions such as: linear (Hillerborg et al 1976), bilinear (Petersson 1981; Wittmann et al 1988; CEP-FIP Model Code 1990), exponential (Gopalaratnam \& Shah 1985; Karihaloo 1995), quasi-exponential (Planas \& Elices 1990) and nonlinear (Reinhardt et al 1986) available in the literature are mathematically expressed below. 


\subsection{Linear softening function}

$$
\left.\begin{array}{ll}
\sigma=f_{t}\left(1-\frac{w}{w_{c}}\right) & \text { for } 0 \leq w \leq w_{c} \\
\sigma=0 & \text { for } w \geq w_{c}
\end{array}\right\}
$$

and

$$
w_{c}=\frac{2 G_{F}}{f_{t}} .
$$

\subsection{Bilinear softening}

The bilinear softening is completely expressed as:

$$
\left.\begin{array}{ll}
\sigma=f_{t}-\left(f_{t}-\sigma_{s}\right) \frac{w}{w_{s}} & \text { for } 0 \leq w \leq w_{s} \\
\sigma=\sigma_{s} \frac{w_{c}-w}{w_{c}-w_{s}} & \text { for } w_{s} \leq w \leq w_{c} \\
\sigma=0 & \text { for } w \geq w_{c}
\end{array}\right\} .
$$

The area under the softening curve is

$$
G_{F}=\frac{\left(\alpha_{s}+\beta_{s}\right)}{2} f_{t} w_{c}
$$

The values of kink point in non-dimensional form are: $\alpha_{s}=\frac{\sigma_{s}}{f_{t}}$, and $\beta_{s}=\frac{w_{s}}{w_{c}}$ where $\sigma_{s}$ and $w_{s}$ are the ordinate and abscissa at the point of slope change of bilinear softening curve. Petersson (1981) used the values of $\alpha_{s}=1 / 3$ and $\beta_{s}=2 / 9$ and hence (26) is expressed as:

$$
w_{c}=\frac{3 \cdot 6 G_{F}}{f_{t}} .
$$

The values of $\alpha_{s}=1 / 4$ and $\beta_{s}=3 / 20$ in bilinear strain-softening diagram were found suitable to simulate the P-COD behaviour of CT specimens (Wittmann et al 1988). In this case the following relation can be obtained.

$$
w_{c}=\frac{5 G_{F}}{f_{t}} .
$$

CEB-FIP Model Code 1990 introduced various empirical relations to obtain the different parameters of bilinear softening curve. In the present program the following relations are used. The values of $w_{c}$ and the constant $k_{d}$ depend on the maximum size of aggregates $\left(d_{a}\right)$ as given below.

\begin{tabular}{|c|c|r|}
\hline$d_{a}(\mathrm{~mm})$ & $w_{c}(\mathrm{~mm})$ & $k_{d}$ \\
\hline 8 & $0 \cdot 12$ & 4 \\
\hline 16 & $0 \cdot 15$ & 6 \\
\hline 32 & $0 \cdot 25$ & 10 \\
\hline
\end{tabular}

For the maximum size of aggregate $19 \mathrm{~mm}$ in present study the values of $w_{c}$ and $k_{d}$ are linearly interpolated as, $w_{c}=0.1688 \mathrm{~mm}$ and $k_{d}=6.75$. The value of $\alpha_{s}=0.15$ is prescribed 
in the CEB-FIP Model Code whereas the value of $\beta_{s}$ for a given value of $G_{F}$ (in $\mathrm{N} / \mathrm{m}$ ) may be obtained using the empirical relations given in the code.

$$
w_{s}=\frac{G_{F}-22 w_{c}\left(\frac{G_{F}}{k_{d}}\right)^{0 \cdot 95}}{150\left(\frac{G_{F}}{k_{d}}\right)^{0 \cdot 95}} \mathrm{~mm}, \& \beta_{s}=\frac{w_{s}}{w_{c}} .
$$

The following relations are obtained using (26) and (29).

$$
\beta_{s}=0.159 \text { and } w_{c}=\frac{6 \cdot 475 G_{F}}{f_{t}} .
$$

\subsection{Exponential softening}

Exponential softening (Gopalaratnam \& Shah 1985) was originally proposed in the following form.

$$
\sigma=f_{t} \exp \left(-k w^{\lambda}\right)
$$

Where, $\lambda$ and $k$ are constant values: $\lambda=1.01$ and $k=0.063$ and $w$ in micrometers. However, in the present study the following exponential relationship (Karihaloo 1995) is used.

$$
\sigma=f_{t} \exp \left(-\mu \frac{w}{w_{c}}\right)
$$

where, $\mu$ is a material constant taken as, $\mu=4.6052$ for $\sigma=0.01 f_{t}$ and $w=w_{c}$, and $w_{c}$ is computed as below.

$$
w_{c}=\frac{4 \cdot 6517 G_{F}}{f_{t}} .
$$

\subsection{Quasi-exponential softening}

Quasi-exponential softening function (Planas \& Elices 1990) is characterized by following expression.

$$
\begin{aligned}
& \sigma(w)=f_{t}\left\{\left(1+c_{1}\right) \exp \left(\frac{-c_{2} w f_{t}}{G_{F C}}\right)-c_{1}\right\} \begin{array}{l}
\text { for } 0<w \leq 5 G_{F C} / f_{t} \\
\text { for } 5 G_{F C} / f_{t} \leq w, \\
\sigma(w)=0 \\
c_{1}=0.0082896, c_{2}=0.96020,
\end{array}
\end{aligned}
$$

where, $c_{1}$ and $c_{2}$ are the material constants.

\subsection{Nonlinear softening}

Nonlinear softening relation (Reinhardt et al 1986) was originally proposed in the following form.

$$
\sigma=f_{t}\left\{\left[1+\left(\frac{c_{1} w}{w_{c}}\right)^{3}\right] \exp \left(\frac{-c_{2} w}{w_{c}}\right)-\frac{w}{w_{c}}\left(1+c_{1}^{3}\right) \exp \left(-c_{2}\right)\right\}
$$




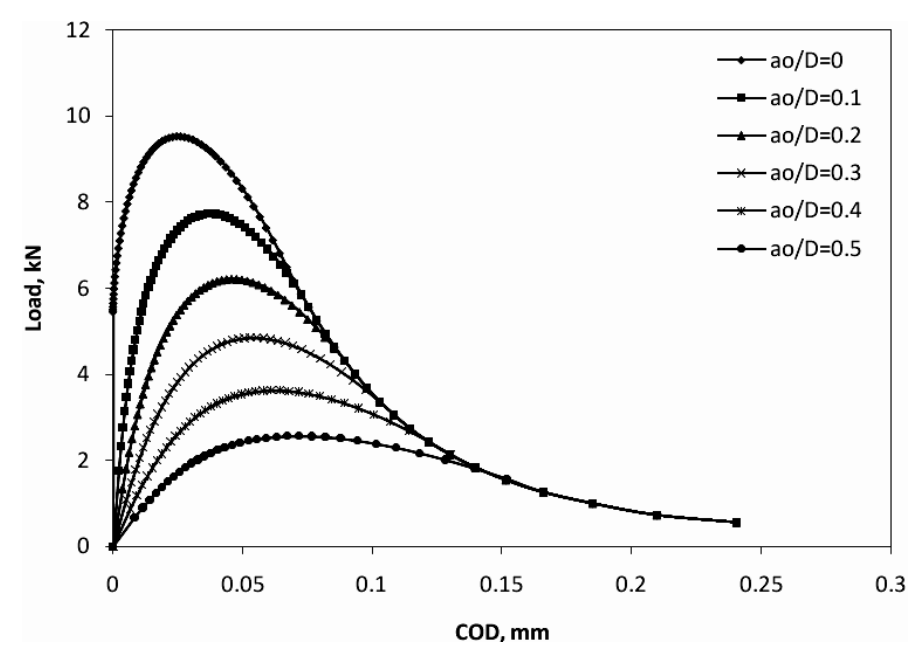

Figure 7. Load-COD curve using linear softening for $D=100 \mathrm{~mm}$.

where, $c_{1}, c_{2}$ and $w_{c}$ and are the material constants. For normal concrete, the three parameters in (35) are considered as $c_{1}=3, c_{2}=6.93, w_{c}=160 \mathrm{~mm}$. Also,

$$
\begin{aligned}
G_{F}= & w_{c} f_{t}\left\{\frac{1}{c_{2}}\left[1+6\left(\frac{c_{1}}{c_{2}}\right)^{3}\right]-\left[1+c_{1}^{3}\left(1+\frac{3}{c_{2}}+\frac{6}{c_{2}^{2}}+\frac{6}{c_{2}^{3}}\right)\right] \frac{\exp \left(-c_{2}\right)}{c_{2}}\right. \\
& \left.-\left(\frac{1+c_{1}^{3}}{2}\right) \exp \left(-c_{2}\right)\right\}
\end{aligned}
$$

$w_{c}$ is computed using the above values of $c_{1}$ and $c_{2}$ and for a given value of $G_{F}$ in (36) as:

$$
w_{c}=\frac{5 \cdot 136 G_{F}}{f_{t}} .
$$

\section{Numerical study}

Three material properties such as modulus of elasticity $E$, uniaxial tensile strength $f_{t}$, and fracture energy $G_{F}$ are required to describe the cohesive crack model. Concrete mix for which, $f_{t}=3.21 \mathrm{MPa}, E=30 \mathrm{GPa}$, and $G_{F}=103 \mathrm{~N} / \mathrm{m}$ (Planas \& Elices 1990) is taken in present investigation. The value of $v$ is assumed to be $0 \cdot 18$. For CT test specimen with thickness $B=100 \mathrm{~mm}$ and size ranging $100 \leq D \leq 600 \mathrm{~mm}$, the finite element analysis is carried out for the study of fracture behaviour. Seven numbers of softening functions as mentioned in previous section are incorporated in the computer program. All calculations are performed with developed computer program using MATLAB for the following numerical investigations.

\subsection{Global P-COD response using linear softening function}

The numerical results of global response of P-COD curves with linear softening are presented in figures 7-9. Figures 7 and 8 are plotted for the $D=100$ and $600 \mathrm{~mm}$ respectively for 


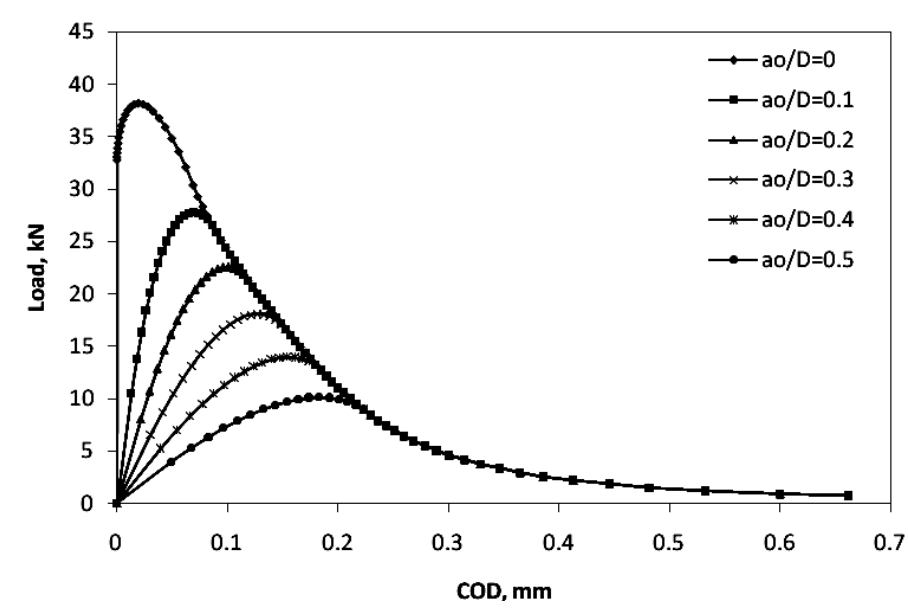

Figure 8. Load-COD curve using linear softening for $D=600 \mathrm{~mm}$.

different initial notch length/depth $\left(a_{o} / D\right)$ ratios ranging from 0 to 0.5 whereas figure 9 is plotted for constant $a_{o} / D$ ratio $=0.3$ for different size range $(100 \leq D \leq 600 \mathrm{~mm})$ specimens. It is observed that the stiffness and the load carrying of the specimen increase with decrease in the value of initial notch-length/depth ratio for a given size or with increase in specimen size for a given value of $a_{o} / D$ ratio. The slope of the softening branch increases by decreasing the value of $a_{o} / D$ ratio for a constant specimen size or by increasing specimen size at a constant value of $a_{o} / D$ ratio. It is also noticed that the uncracked specimen becomes more unstable after the peak load is reached whereas the specimen behaves in more ductile manner and better controllable as the $a_{o} / D$ ratio increases for a specified specimen size or the specimen size decreases at constant value of $a_{o} / D$ ratio for cracked specimen. The terminal branch of the P-COD curves appears to be converging and is independent of the $a_{o} / D$ ratio for a given size specimen which is caused by the assumption made in the analysis that the damage zone has zero thickness and concentrated on a line. Measuring P-COD curve,

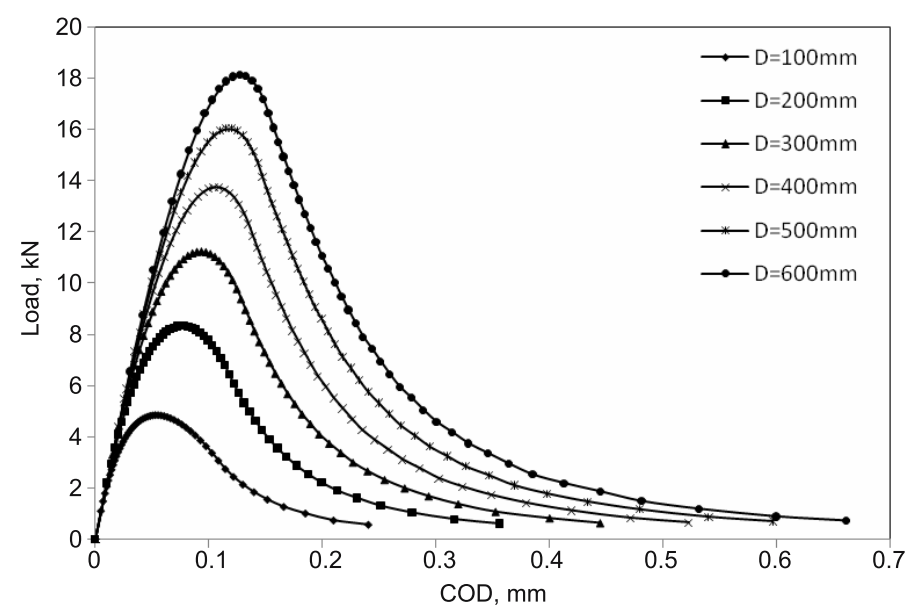

Figure 9. Load-COD curve using linear softening for $100 \leq D \leq 600 \mathrm{~mm}$ and $a_{o} / D=0 \cdot 3$. 
the snap-back instability behaviour shown in case of $\mathrm{P}-\delta$ (load-deflection) curve of TPBT specimen is avoided because in the P-COD curve, the crack opening displacement increases monotonically in softening zone during crack growth.

The cohesive crack behaviour as discussed above and demonstrated in figures 7 and 8 may be practically different, if the actual width of fracture process zone and the crack path are modelled accurately. Because, during crack propagation in concrete, a macro-crack has a tendency to follow a tortuous crack path due to various mechanisms of micro-cracking, crack bridging, aggregate interlocking, etc. The meandering of main crack depends on the concrete mix design and the properties of the aggregates particles such as size, texture and angularity. The fracturing topography of concrete is complicated because of its complex heterogeneity. Issa et al $(1993,2003)$ characterized the fracture surfaces of concrete using simple nondestructive modified slit-island experimental technique. They showed that the main crack can deflect around the aggregates and or penetrates through the aggregates depending the strength of cement paste and the aggregates location with respect to the crack front. This phenomenon results in the energy consumption and affects the apparent toughness of the material. It was also observed that the fracture surfaces of concrete appeared to be relatively rougher for the larger size aggregates than those for the smaller size aggregates. Fracture tests and analysis on wedge-splitting concrete specimens of different sizes, made with varying sizes of aggregates (Issa et al 2000a; 2000b) revealed that even no two identical specimens show the same crack path and for the similar concrete specimens, the crack tortuosity is greater for the larger size specimens. The cohesive crack model is a strictly uniaxial method considering zero width of the process zone and it does not allow the crack path to follow in an actual tortutous way. The assumptions made in the development of the model is differed from the experimentally observed real behaviour of fracture process of concrete and thus it allows the terminals of the softening branches to converge irrespective of the relative initial-notch lengths.

\subsection{Influence of softening functions on the global P-COD response}

The results of P-COD curves using various shapes of softening functions for compact tension specimens with $a_{o} / D$ ratio $=0.3$ are determined and presented in figures 10 and 11 for $D=100 \mathrm{~mm}$ and $600 \mathrm{~mm}$, respectively.

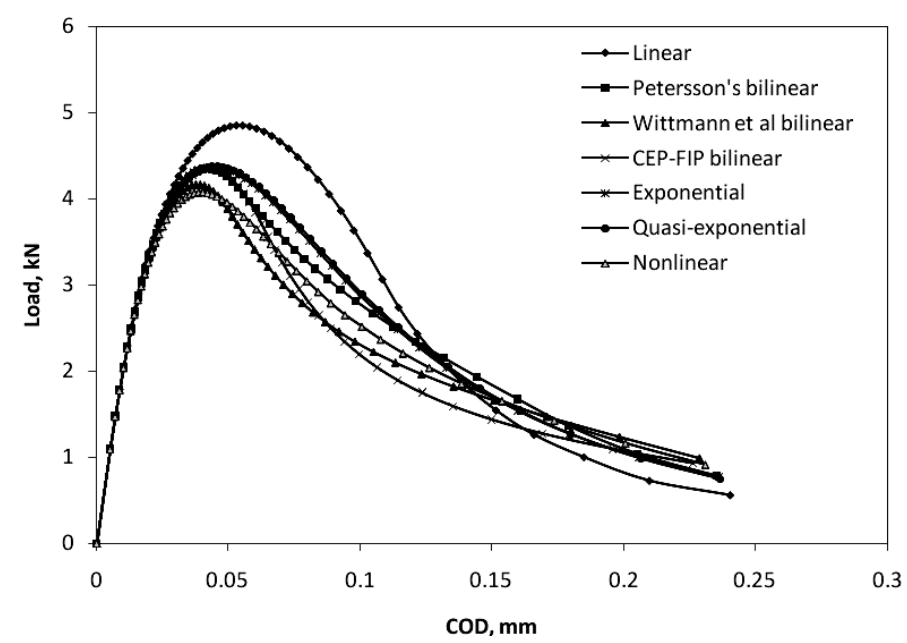

Figure 10. Influence of softening curve on Load-COD curve for $D=100$ and $a_{o} / D=0 \cdot 3$. 


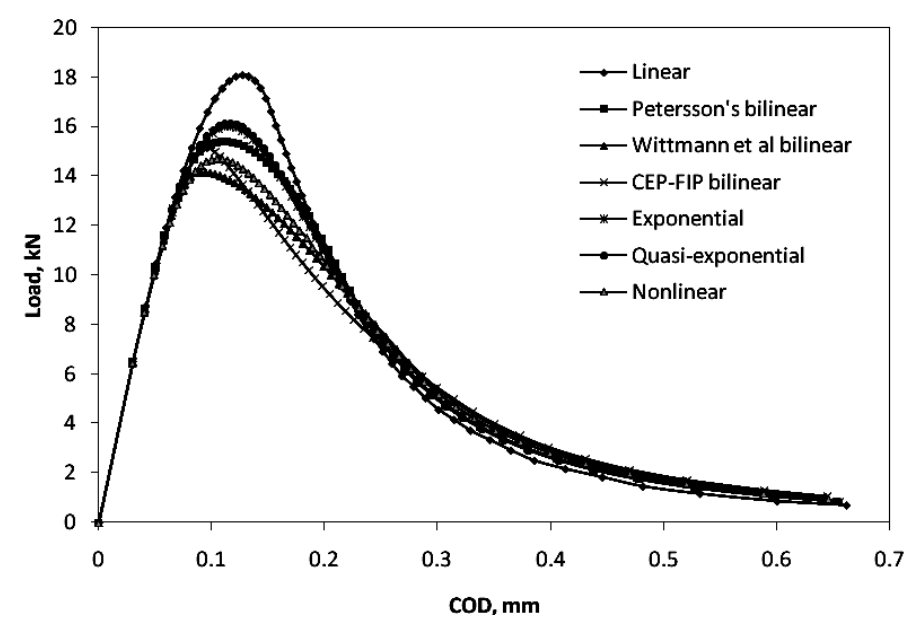

Figure 11. Influence of softening curve on Load-COD curve for $D=600$ and $a_{o} / D=0 \cdot 3$.

The peak nominal stresses $\left(P_{u} / B D\right)$ obtained using cohesive crack model with aforementioned six softening relations (except for linear softening) for 100 and $600 \mathrm{~mm}$ size specimens are in the range of 0.407-0.438 MPa and 0.245-0.268 MPa respectively showing a less scatter in results and the difference between minimum and maximum peak nominal stresses for all sizes of specimens is less than $7.07 \%$ and $8.58 \%$ respectively. The minimum and maximum nominal peak stresses are yielded by nonlinear softening and quasi-exponential softening functions respectively for the both size range specimens. The peak nominal stress using linear softening function for 100 and $600 \mathrm{~mm}$ size specimens are 0.485 and $0.302 \mathrm{MPa}$ respectively. On an average the linear softening yields the peak load $11.75 \%$ and $15.89 \%$ larger than the bilinear curves, exponential, quasi-exponential and nonlinear softening functions for the size range of 100 and $600 \mathrm{~mm}$ respectively. The post-peak response of P-COD curves with different softening curves (except linear softening) is slightly varied. Almost the same P-COD curves can be obtained using exponential and quasi-exponential softening functions.

\subsection{Influence of softening functions on the size-scale transition towards LEFM}

A non-dimensional parameter known as brittleness number $(\beta)$ for geometrically similar structures is used for the study of nonlinear fracture behaviour.

$$
\beta=\frac{D}{l_{c h}} .
$$

The numerical results with varying values of $\beta$ are obtained to show the deviation from LEFM concept for geometrically similar normal size CT specimens having $B=100 \mathrm{~mm}$, the material properties $G_{F}=103 \mathrm{~N} / \mathrm{m}$ and $f_{t}=3.21 \mathrm{MPa}$ using all seven softening functions. The maximum load using cohesive crack model $\left(P_{u, C C M}\right)$ or true peak load and using well known LEFM formula $\left(P_{u, L E F M}\right)$ are computed at initial crack length/depth ratio such as $a_{o} / D=0 \cdot 3$. The LEFM formula (ASTM Standard 399 2006) for CT specimen is used to calculate the critical value of stress intensity factor $K_{I C}$ for known value of fracture energy 
$G_{I C}$ as mentioned below:

$$
\begin{aligned}
& K_{I C}=\sigma_{N u} \sqrt{D} k(\alpha) \\
& k(\alpha)=\frac{(2+\alpha)\left[0 \cdot 886+4 \cdot 64 \alpha-13 \cdot 32 \alpha^{2}+14 \cdot 72 \alpha^{3}-5 \cdot 6 \alpha^{4}\right]}{(1-\alpha)^{3 / 2}} .
\end{aligned}
$$

Where; $\alpha=\frac{a}{D}=\alpha_{o}=\frac{a_{o}}{D}, \sigma_{N u}=\frac{P}{B D}$ and (40) is valid for $0.2 \leq \alpha \leq 1$ within $0.5 \%$ accuracy. The maximum nominal stress due to external load $P_{u, L E F M}$ of the structure is determined by: $P_{u, L E F M}=\sigma_{N u} B D$. Neglecting the effect of Poisson's ratio, the value of $K_{I C}$ is evaluated using following relation.

$$
K_{I C}=\sqrt{G_{I C} E} .
$$

The result of the ratio $P_{u, C C M} / P_{u, L E F M}$ with varying values of $\beta$ is plotted in figure 12 . The ratio of $P_{u, C C M} / P_{u, L E F M}$ may be considered as the ratio of the fictitious fracture toughness determined using true peak load to the true fracture toughness (as a material constant). From the figure it is observed that the peak load predicted using LEFM is normally overestimated as compared to the peak load (or true peak load) obtained using cohesive crack model. This deviation is higher at low value of brittleness number $\beta$ whereas for high $\beta$ values the results of the cohesive crack model tend to be the same as those predicted by LEFM i.e., $P_{u, C C M} \approx P_{u, L E F M}$ for $\beta \rightarrow \infty$. This infers that the peak load obtained using LEFM deviates from the true peak load obtained using CCM for normal size of concrete elements. According to the Report (Petersson 1981), the LEFM is applicable to concrete in case the value of $\beta$ is more than 10. For normal concrete, the value of $l_{c h}$ normally varies between 200 and $300 \mathrm{~mm}$, which implies that LEFM is applicable only when the beam depth is in between 2 to $3 \mathrm{~m}$. It is also pointed out (Bažant 2002) that the length of FPZ in normal concrete is in the order of $500 \mathrm{~mm}$ and depending upon the structure size $D$ different fracture mechanics concepts might be appropriate for analysing the failure behaviour. The classical form of LEFM may be approximately suitable for the ratio $D / F P Z \geq 100$ meaning that the LEFM might be applied for normal concrete structures for $D \approx 50 \mathrm{~m}$.

Figure 12 further shows that the predicted ratio $P_{u, C C M} / P_{u, L E F M}$ using linear softening is higher than those using other six softening functions for the considered size range and it seems

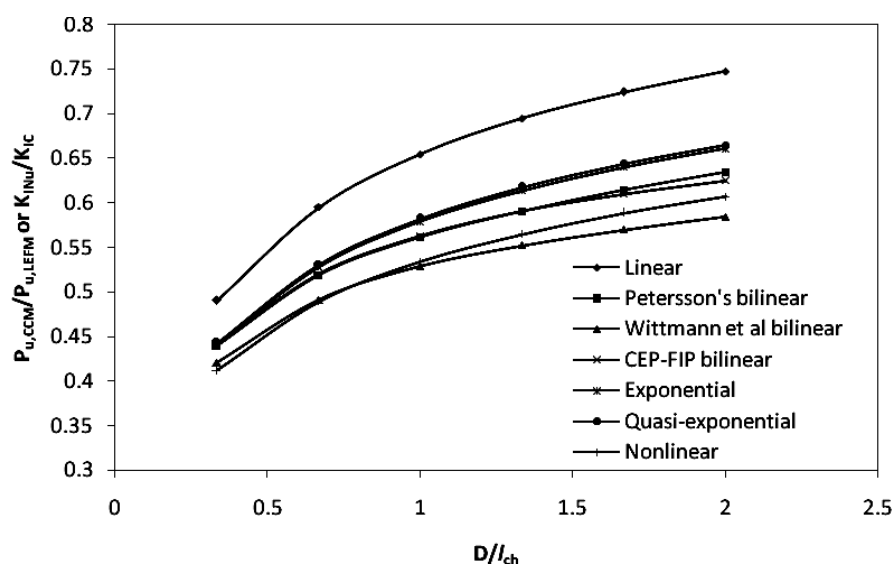

Figure 12. Influence of softening curve on size-scale transition towards LEFM for $a_{o} / D=0 \cdot 3$. 


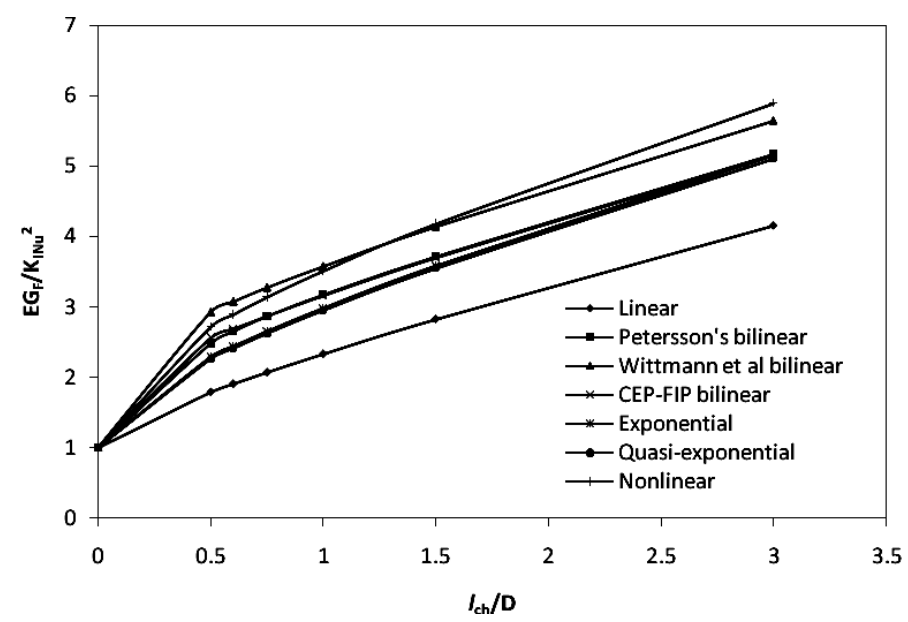

Figure 13. Influence of softening curve on size-effect curve for $a_{o} / D=0 \cdot 3$.

that the difference is more diverged as the size-scale increases. On the other hand, the ratio of $P_{u, C C M} / P_{u, L E F M}$ for the bilinear curves, exponential, quasi-exponential and nonlinear functions is relatively confined in narrow band for lower specimen size and seems to be slightly diverging for higher size-scale. However, this ratio is almost the same for the exponential and quasi-exponential and also for Petersson's bilinear and CEB-FIP bilinear softening functions. The minimum and maximum values of the ratios $P_{u, C C M} / P_{u, L E F M}$ for all the softening functions (except linear) is in the range of 0.412-0.443 (for nonlinear softening) (for quasiexponential softening) for $D=100 \mathrm{~mm}$ and in the range of 0.584-0.665 (for Wittmann et al bilinear softening) (for quasi-exponential softening) for $D=600 \mathrm{~mm}$ respectively. Moreover, the ratio $P_{u, C C M} / P_{u, L E F M}$ for linear softening is 0.491 for $D=100 \mathrm{~mm}$ and 0.748 for $D=600 \mathrm{~mm}$ respectively.

\subsection{Influence of softening functions on the size-effect curve}

The size-effect is defined as the decrease in nominal strength of geometrically similar structures subjected to symmetrical loads when the characteristic size of the structure is increased. There are two extremes of size-effect law: (i) strength criteria and (ii) LEFM size-effect. The former yields no size-effect whereas the latter shows the strongest size effect i.e. nominal strength is inversely proportional to the square root of the structural dimension. Quasi-brittle materials like concrete exhibit a transitional size effect between the two extremes of sizeeffects. The generalized size effect law using cohesive crack model can be expressed in the following form (Planas \& Elices 1990).

$$
\frac{E G_{F}}{K_{I N u}^{2}}=1+C_{1}\left(\frac{l_{c h}}{D}\right)+C_{2}\left(\frac{l_{c h}}{D}\right)^{2}+C_{3}\left(\frac{l_{c h}}{D}\right)^{3}+\cdots,
$$

where, $C_{i}=$ dimensionless coefficients depending on geometry and material properties, $K_{I N u}=$ stress intensity factor computed for maximum actual loads, $P_{u}$ and initial crack length. The left hand terms and $l_{c h} / D$ of right hand terms represent $Y$ and $X$ co-ordinates respectively on the plot size-effect curve using (42). This equation is made to satisfy in such a manner that CCM and LEFM will yield the same result for infinitely large structures 
$(D \rightarrow \infty)$. The size-effect curves are plotted in non-dimensional forms of inverse of nominal stress intensity factor, $K_{I N u}$ at peak load vs. inverse of characteristic dimension $D$ at constant $a_{o} / D$ ratio of $0 \cdot 3$ as shown in figure 13. It is observed from the figure that the size-effect curves are in general influenced by the softening function of concrete. The figure further shows that the predicted ratio $E G_{F} / K_{I N u}^{2}$ using linear softening is lower than those using other six softening functions for the considered size range and the ratio of $E G_{F} / K_{I N u}^{2}$ for the bilinear curves, exponential, quasi-exponential and nonlinear functions are relatively less varied. Almost the same size-effect curves can be obtained using exponential, quasi-exponential softening functions and also using Petersson's bilinear and CEB-FIP bilinear softening functions. It is also seen from the figure that as the size increases all size-effect curves seem to be converging and for infinitely large structures $(D \rightarrow \infty)$ the values of $E G_{F} / K_{I N u}^{2}$ tend asymptotic to be 1 exhibiting that fracture loads predicted by LEFM and CCM are the same for all size-effect curves.

\subsection{Evolution of fracture zone}

Development of fracture zone and stress distribution ahead of the initial crack-tip of a CT specimen for $D=600 \mathrm{~mm}$ and $a_{o} / D$ ratio $=0.3$ with Wittmann et al (1988) bilinear softening function are shown in figure 14 for four salient positions of load on the P-COD curve. In the figure, the development of fracture zone is shown by filled-up solids. The length of fracture zone and the stress distribution ahead of the crack-tip are represented (not to scale) for explaining the mechanism of process zone evolution. The fracture zone begins to form as soon as the structure is loaded. When the load position is approximately at 1 , the fracture zone is small and the developed stress value in the process zone is always less than the tensile strength $f_{t}$ of the material. This stress distribution does not show any stress singularity unlike in LEFM analysis. When the load position is at 2 i.e. at peak load, the length of fracture zone becomes about $75 \mathrm{~mm}$ and the stress value at the initial crack-tip $\sigma_{\text {tip }}$ achieves a value of $0.2423 f_{t}$. The lengths of fracture zone corresponding to peak load and fully developed FPZ obtained using Wittmann et al (1988) bilinear softening function for specimen size range $100-600 \mathrm{~mm}$ are presented in figure 15 . It can be seen that fracture zone at peak load for $D=100 \mathrm{~mm}$ and $a_{o} / D$ ratio $=0.3$ becomes about $28.75 \mathrm{~mm}$. In addition, the material is still able to transfer stress across the crack faces even up to peak load and further loading of structure corresponding to load position 3 , the stress transferring capacity of concrete becomes to zero. At this stage of loading, a full length of fracture zone i.e. FPZ can be observed. For $D=600 \mathrm{~mm}$, the length of FPZ is about $300 \mathrm{~mm}$ and is significant and variable with respect to specimen size as shown in figure 15 . The fully developed FPZ for specimen size $D=100 \mathrm{~mm}$ is missing in the figure because it is not developed up to $78^{\text {th }}$ node in the potential fracture line. Further loading of structure say position 4, the stress-free crack is generated and the real crack has moved to a distance say $\Delta a$.

It is observed that the length of fracture zone at peak load and FPZ vary with the specimen size significantly. As shown in figure 15, the fracture zones increase with increase in specimen size. However, the relative size of the fracture zone with respect to the specimen size decreases with increasing specimen size. This infers that the fracture behaviour of normal concrete depends on the specimen size. For limiting case of infinitely large structure, the relative sizes of fracture zone and FPZ become very small and insignificant such that the LEFM can be applied. In general, it can be stated that the local and global responses during crack initiation and its growth of cementitious materials are dependent on many factors. Hence, the influence of softening function and size-effect on the important fracture parameters are presented in subsequent analyses. 

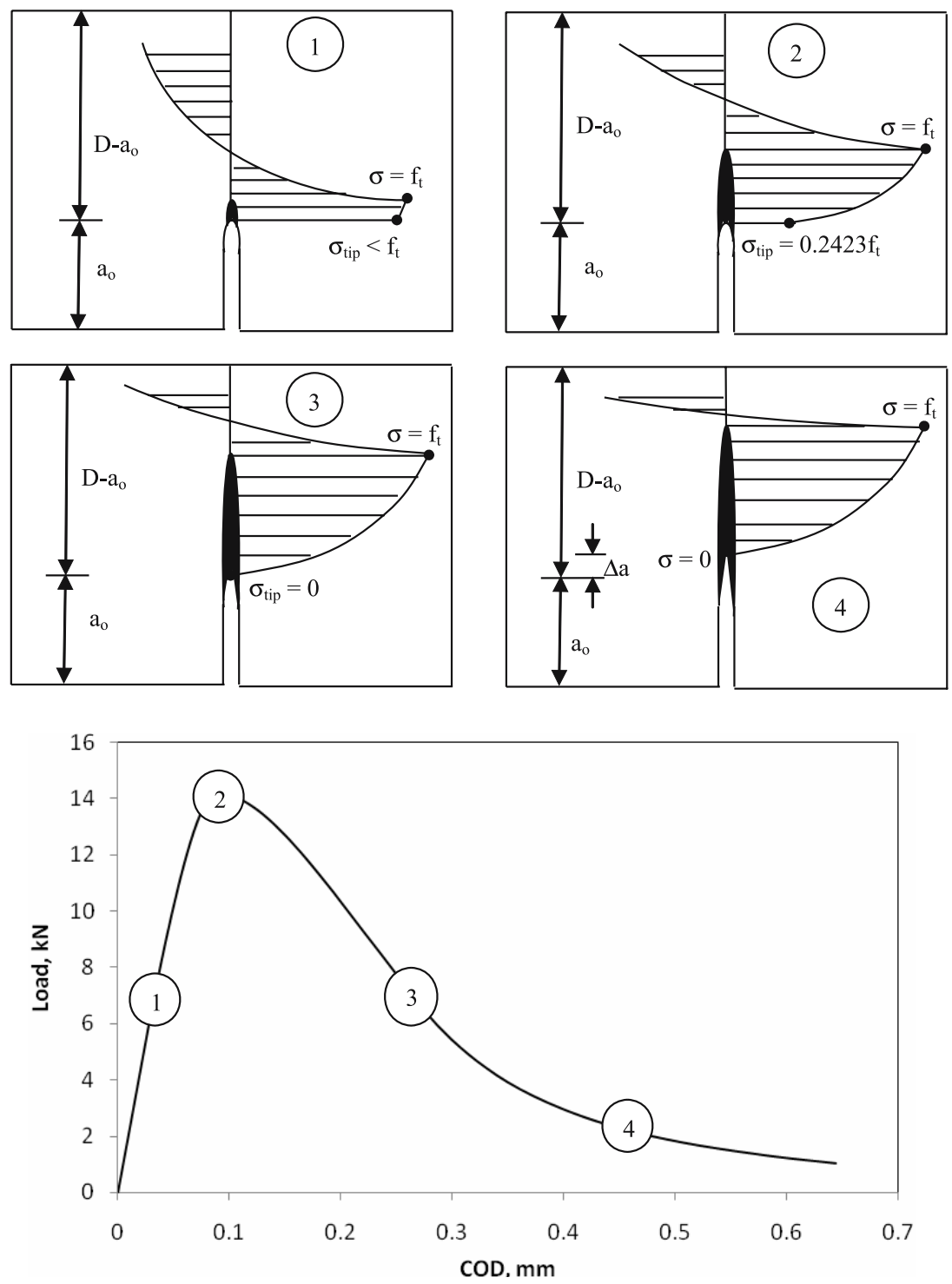

Figure 14. Evolution of fracture zone and stress distribution ahead of the crack-tip for different positions of load on the P-COD curve obtained from Wittmann et al (1988) bilinear softening for $D=600 \mathrm{~mm}$ and $a_{o} / D=0 \cdot 3$.

\subsection{Influence of softening functions on the development of fracture process zone}

The length of fully developed fracture process zone is computed using all seven softening functions for the specimen size-range $200 \leq D \leq 600 \mathrm{~mm}$ because full length of FPZ is not developed even up to 78 nodes using some of the softening functions for $100 \mathrm{~mm}$ size specimen. The length of FPZ/depth (FPZ/D) ratio shows a definite relationship with the brittleness of the structures as shown in figure 16; however, it also depends on the shape of the softening relations used. Almost the same prediction of FPZ is seen using exponential or quasi-exponential softening functions of concrete. The length of FPZ obtained using the linear 


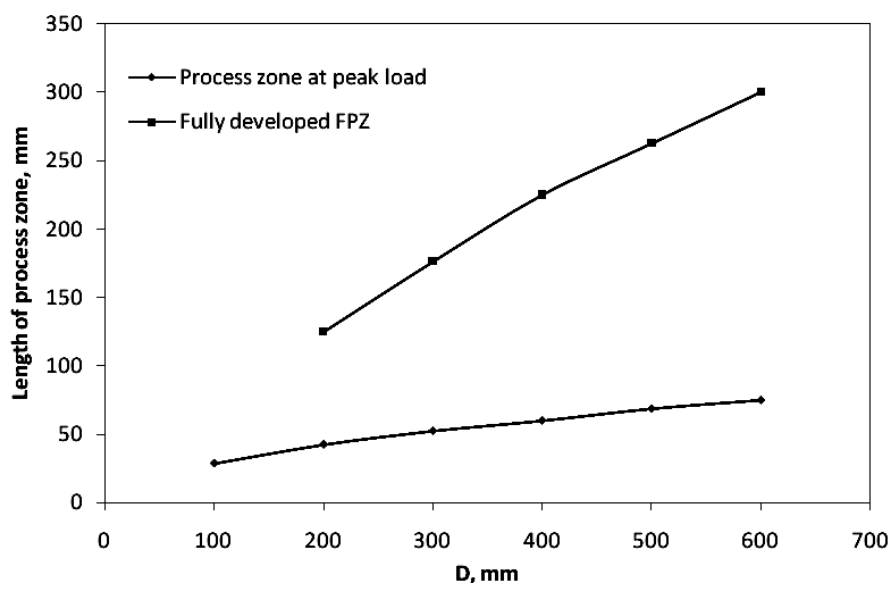

Figure 15. Depth of fracture zone at peak load and fully developed FPZ for $a_{o} / D=0 \cdot 3$ obtained using Wittmann et al (1988) bilinear softening function.

softening is the minimum as compared to those obtained using bilinear curves, exponential, quasi-exponential and nonlinear functions for all size-range. It is evident from the figure that the relative size of fracture process zone decreases as the size of specimen is increased which is the main source of size-effect in quasibrittle materials. The minimum and maximum values of the ratio FPZ/D for all the softening functions (except linear) is in the range of 0.575-0.65 (for Petersson's bilinear softening) (for CEB-FIP bilinear softening) for $D=200 \mathrm{~mm}$ and in the range of $0 \cdot 4-0.563$ (for Petersson's bilinear softening) (for CEB-FIP bilinear softening) for $D=600 \mathrm{~mm}$ respectively. The difference between minimum and maximum fully developed FPZ is less than $11.54 \%$ and $28.95 \%$ for $\mathrm{D}=200 \mathrm{~mm}$ and $600 \mathrm{~mm}$ respectively. The ratio FPZ/D for linear softening is 0.438 for $D=200 \mathrm{~mm}$ and 0.238 for $D=600 \mathrm{~mm}$ respectively. On an average the linear softening yields the FPZ $29.22 \%$ and $51.38 \%$ lower than the bilinear curves, exponential, quasi-exponential and nonlinear softening functions for the size range $200 \mathrm{~mm}$ and $600 \mathrm{~mm}$ respectively.

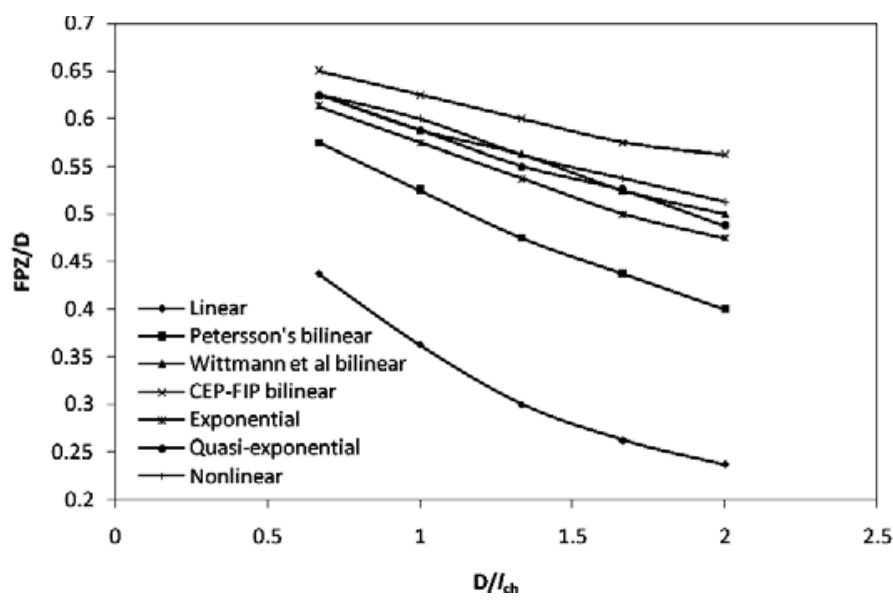

Figure 16. Influence of softening curve on fully developed FPZ for $a_{o} / D=0 \cdot 3$. 


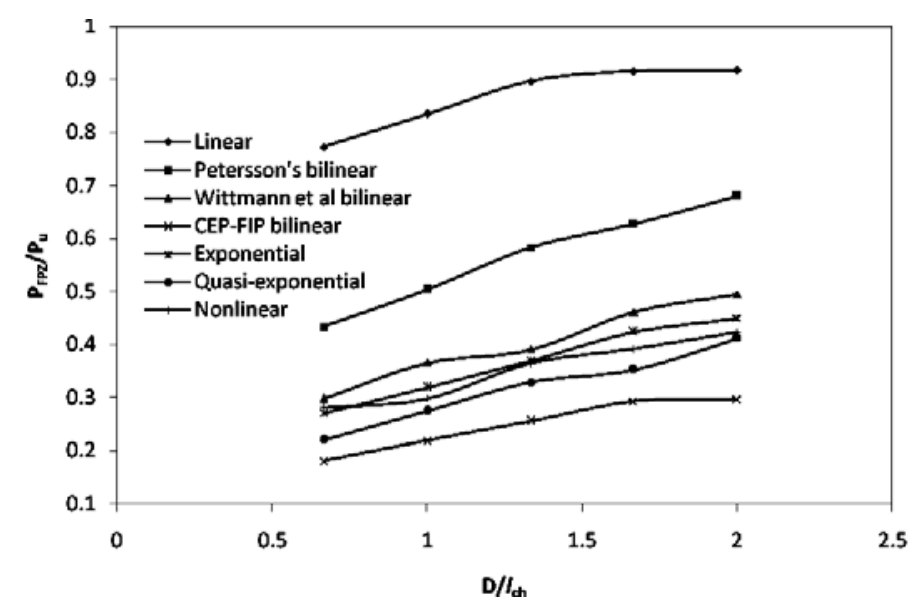

Figure 17. Influence of softening curve on the ratio of load $P_{F P Z} / P_{u}$ for $a_{o} / D=0 \cdot 3$.

\subsection{Influence of softening functions on the load carrying capacity corresponding to the fully developed FPZ}

The load corresponding to fully developed fracture process zone $P_{F P Z}$ is calculated for the specimen size-range $200 \leq D \leq 600 \mathrm{~mm}$ using all the softening functions and results are plotted in the non-dimensional ratio of $P_{F P Z} / P_{u}$ with respect to the brittleness of the structures as shown in figure 17. It is clear from the figure that the ratio of $P_{F P Z} / P_{u}$ is a increasing function with the brittleness of quasibrittle structures. It implies that the value of $P_{F P Z} / P_{u}$ increases as the size of specimen increases. It is evident that the larger is the value of $P_{F P Z} / P_{u}$ on the P-COD curve, the closer is the load corresponding to fully developed FPZ to the peak load in softening zone. Cohesive crack model thus illustrates the variation from failure at relatively larger fracture process zone for small structures to the failure at a relatively smaller fracture process zone for large structures. The minimum and maximum values of the ratios $P_{F P Z} / P_{u}$ for all the softening functions (except linear) is in the range of 0.219-0.433 (for CEB-FIP bilinear softening) (for Petersson's bilinear softening) for $D=200 \mathrm{~mm}$ and in the range of 0.297-0.680 (for CEB-FIP bilinear softening) (for Petersson's bilinear softening) for $D=600 \mathrm{~mm}$ respectively. The ratio $P_{F P Z} / P_{u}$ for linear softening is 0.773 for $D=200 \mathrm{~mm}$ and 0.918 for $D=600 \mathrm{~mm}$ respectively. This means that the length of fully developed FPZ using linear softening is always smaller when compared to those predicted using bilinear curves, exponential, quasi-exponential and nonlinear functions.

\subsection{Influence of softening functions on the fictitious crack length corresponding to peak load}

Fictitious crack length/depth $\left(a_{f} / D\right)$ ratio at peak load is obtained using all seven softening functions and the variation of $a_{f} / D$ ratio is plotted with brittleness of concrete as shown in figure 18. It is observed from the figure that the $a_{f} / D$ ratio at peak load maintains a definite relationship with brittleness of the structure and the influence of the shape of softening functions including linear one on the value of $a_{f} / D$ ratio is relatively less. The relationship shows that for low brittleness the fictitious crack length at peak load is covered in almost whole ligament resulting in a ductile failure, on the other hand, the process zone decreases with increase in brittleness and it appears that $a_{f} / D \rightarrow a_{o} / D$ for $D / l_{c h} \rightarrow \infty$. The minimum 


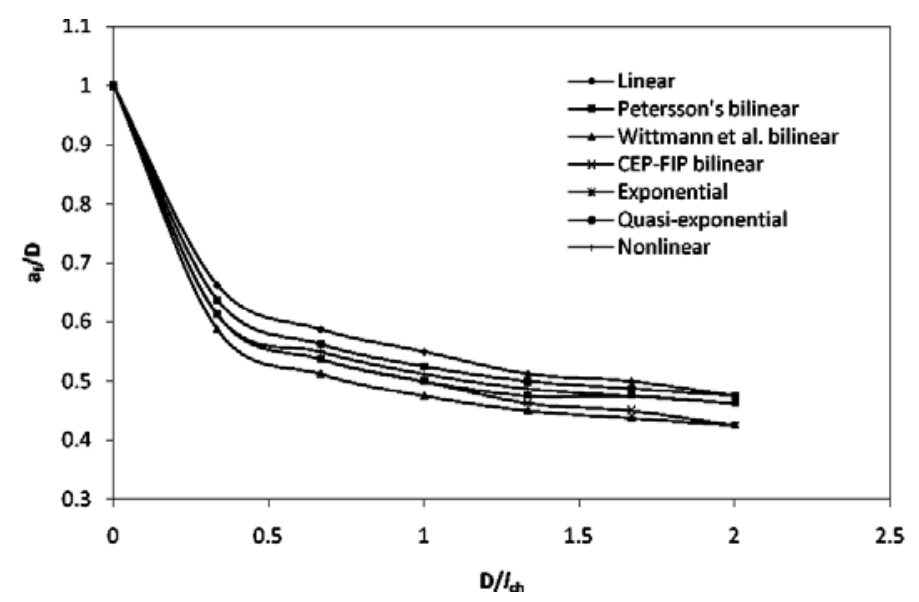

Figure 18. Influence of softening curve on the fictitious crack length/depth $\left(a_{f} / D\right)$ ratio for $a_{o} / D=0 \cdot 3$.

and maximum values of $a_{f} / D$ ratio for all the softening functions is in the range of $0.588-$ 0.663 (for Wittmann et al bilinear) (for linear softening) for $D=100 \mathrm{~mm}$ and in the range of 0.425-0.475 (for Wittmann et al bilinear and CEB-FIP bilinear) (for linear softening) for $D=600 \mathrm{~mm}$ respectively.

\subsection{Influence of softening functions on the CTO $D_{c}$}

Critical value of crack-tip opening displacement $\left(C T O D_{c}\right)$ corresponding to peak load is measured from the numerical results of cohesive crack model and plotted using each softening function as shown in figure 19. In predicting the $C T O D_{c}$ using CEB-FIP model code (1990) bilinear softening, some oscillations are noticed beyond the specimen size of $300 \mathrm{~mm}$. It is observed from the figure that in general the value of $C T O D_{c}$ is a function of brittleness and depends upon the shape of softening function of concrete except for the exponential and

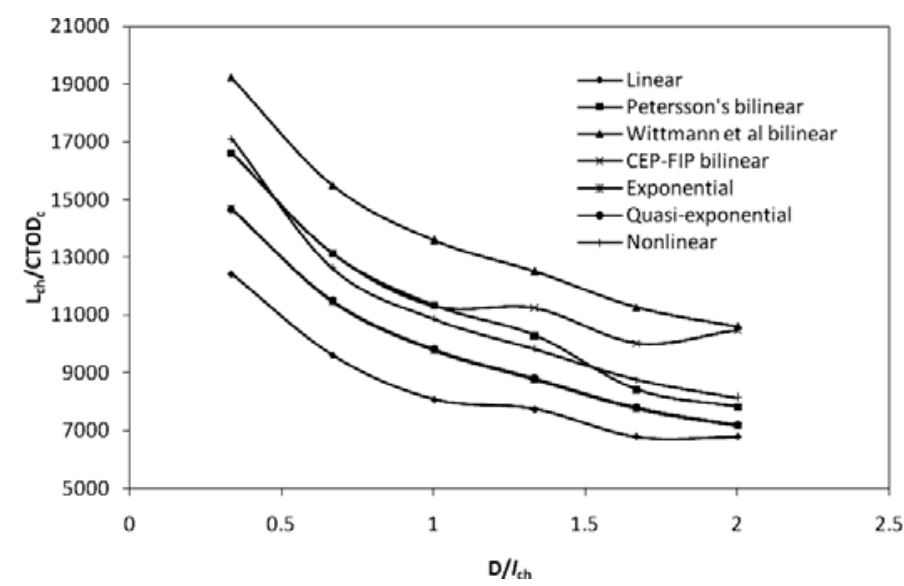

Figure 19. Influence of softening curve on the $C T O D_{c}$ for $a_{o} / D=0.3$. 


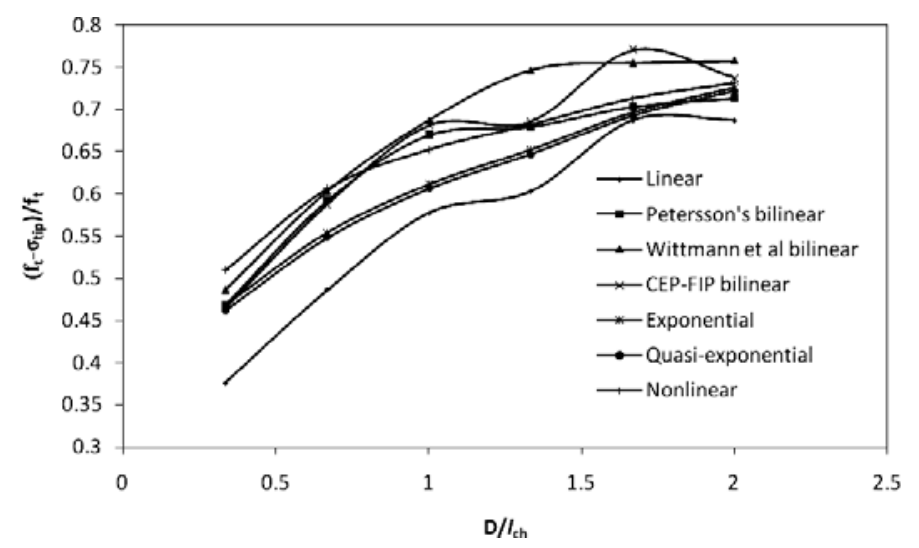

Figure 20. Influence of softening curve on crack tip stress at peak load for $a_{o} / D=0 \cdot 3$.

quasi-exponential softening functions which yield almost the same values of $C T O D_{c}$ for all size specimens. The value of $C T O D_{c}$ increases as the specimen size increases meaning that the relative size of FPZ will be smaller for higher brittleness of the structures. The CTOD obtained using linear softening is always greater than those obtained using bilinear curves, exponential, quasi-exponential and nonlinear functions for all the size-range. The minimum and maximum values of the ratios $l_{c h} / C T O D_{c}$ for all the softening functions (except linear) is in the range of 14668-19220 (for quasi-exponential softening) (for Wittmann et al softening) for $D=100 \mathrm{~mm}$ and in the range of 7163-10613 (for quasi-exponential softening) (for Wittmann et al softening) for $D=600 \mathrm{~mm}$ respectively. The ratio $l_{c h} / C T O D_{c}$ for linear softening is 12428 for $D=100 \mathrm{~mm}$ and 6802 for $D=600 \mathrm{~mm}$ respectively.

\subsection{Influence of softening functions on the local stress distribution at notch-tip correspond- ing to peak load}

Influence of shape of softening curve and size-effect on the notch-tip stress $\sigma_{t i p}$ distribution corresponding to peak load is shown in figure 20. The ordinate of figure is the value of $\left(f_{t}-\sigma_{t i p}\right) / f_{t}$ which may be termed as tip stress transfer ratio and the abscissa is the $D / l_{c h}$. It is evident that for $\sigma_{t i p}=f_{t}$ i.e. the fracture node at notch-tip is not yet opened the tip stress is equal to the tensile strength of the material and for the other extreme the fracture process zone is fully developed when the notch-tip stress $\sigma_{t i p}=0$. Therefore value of the tip stress transfer ratio ranges between 0 and 1 (when the notch-tip has not yet opened) (when FPZ is fully developed and any further loading will result in stress free crack propagation). From the figure it is observed that the values of $\left(f_{t}-\sigma_{t i p}\right) / f_{t}$ depend on the shape of the softening curve and the specimen size. Similar to figure 19, with the use of CEB-FIP Model Code (1990) bilinear and linear softening functions, some degrees of oscillations are observed in figure 20. Within the size-range considered in the study, these oscillations are more pronounced beyond the specimen sizes about $300 \mathrm{~mm}$ which may be possibly due to increasing specimen size and consequently resulting in the increased distance between the cohesive nodal forces. In general, there is an increasing trend in the value of the ordinates $\left(f_{t}-\sigma_{t i p}\right) / f_{t}$ as the specimen size increases. It shows that the size of fracture zone is influenced by the specimen size and shape of the softening relation used in the cohesive crack model. It is also observed that the stress transferring capacity at the crack-tip corresponding to the peak load decreases as the specimen 
size increases and as a result of which a real crack begins to propagate closer to the peak load for larger size specimens. It is also clear that the slow crack growth does not commence before the softening damage takes place. This behaviour predicted using exponential and quasi-exponential softening functions is almost the same. The minimum and maximum values of $\left(f_{t}-\sigma_{t i p}\right) / f_{t}$ for all the softening functions (except linear) is in the range of $0.462-$ 0.510 (for quasi-exponential softening) (for nonlinear softening) for $D=100 \mathrm{~mm}$ and in the range of 0.732-0.758 (for quasi-exponential softening) (Wittmann et al bilinear softening) for $D=600 \mathrm{~mm}$ respectively. The ratio $\left(f_{t}-\sigma_{t i p}\right) / f_{t}$ obtained using linear softening is 0.376 for $D=100 \mathrm{~mm}$ and 0.687 for $D=600 \mathrm{~mm}$, respectively. It is revealed that the mean tip stress transfer ratio considering all softening functions (except linear) is 0.474 and 0.732 for $D=100 \mathrm{~mm}$ and $D=600 \mathrm{~mm}$ respectively which are always greater than those predicted using linear softening relation.

\section{Comparison with experimental result}

Compact tension specimens of different sizes made of various mix proportions and water/cement (W/C) ratios were tested by Wittmann et al (1988) under three different loading rates. The tests were conducted on the $\mathrm{CT}$ specimens having measured standard dimensions of $a_{o}$ is $0.5 \mathrm{D}, D_{1}$ is $1.25 \mathrm{D}, \mathrm{H}$ is $0.6 \mathrm{D}$ and $H_{1}$ is $0.5 \mathrm{H}$. In which, the dimensions $D$ and $H_{1}$ are not indicated in the figure (Wittmann et al 1988) and they are carefully measured and found to be $2 a_{o}$ and $0.5 \mathrm{H}$ respectively. Three sizes of specimens $D=300,600$ and $1200 \mathrm{~mm}$ with constant thickness $B=120 \mathrm{~mm}$ were tested and the mean curves of load-crack opening displacement in the plane of loading of loading points (P-COD) for specimens $D=600$ and $1200 \mathrm{~mm}$ and load-crack mouth opening displacement (P-CMOD) for specimens of $D=300 \mathrm{~mm}$ were measured. The medium size of CT specimens, $D=600 \mathrm{~mm}$ made of concrete with W/C ratio 0.43 and maximum aggregate size of $16 \mathrm{~mm}$ was considered as the reference specimens. The reference specimens were tested under loading rate of $0.2 \mathrm{~mm} / \mathrm{min}$ for which the 28-day compressive strength of concrete was determined as $42.9 \mathrm{MPa}$. From fracture tests, the mean value of peak load and the specific fracture energy were obtained as $12.6 \mathrm{kN}$ and $162 \mathrm{~N} / \mathrm{m}$ respectively. In order to calculate the essential parameters of a bilinear softening function using fictitious crack model, a method was also suggested by the authors (Wittmann et al 1988). Besides the other important studies, it was concluded that load-displacement curves can be determined by means of finite element analysis with the assumption of bilinear softening functions and are in a good agreement with those experimentally observed curves. The specific fracture energy can thus be calculated from the strain-softening relation that agrees well to the same determined directly from the P-COD curve. As such, the authors (Wittmann et al 1988) suggested that the parameters of the bilinear softening function as $G_{F}=157 \mathrm{~N} / \mathrm{m}$ and $f_{t}=4.4 \mathrm{MPa}$ can predict the fracture behaviour of reference specimen in a better manner.

In the present work, a realistic comparison between the experimental and numerical results is carried out for the application of various softening functions in the cohesive crack model. For the numerical modelling, a concrete mix having maximum size of aggregate $d_{a}=16 \mathrm{~mm}$ and its properties $v=0.18$ and $G_{F}=157 \mathrm{~N} / \mathrm{m}$ is considered. The value of $E$ is obtained as 34.9 GPa using CEB-FIP Model Code (1990) for a given value of compressive strength $42.9 \mathrm{MPa}$. A similar the finite element discretization of the $\mathrm{CT}$ specimen as shown in figure 6 is done for determining the influence coefficients in which the relation: $H_{1}=0.5 \mathrm{H}$ is considered to simulate the actual test specimen. All the seven softening functions are applied to determine the P-COD curves for the comparison with the mean P-COD curve obtained from 


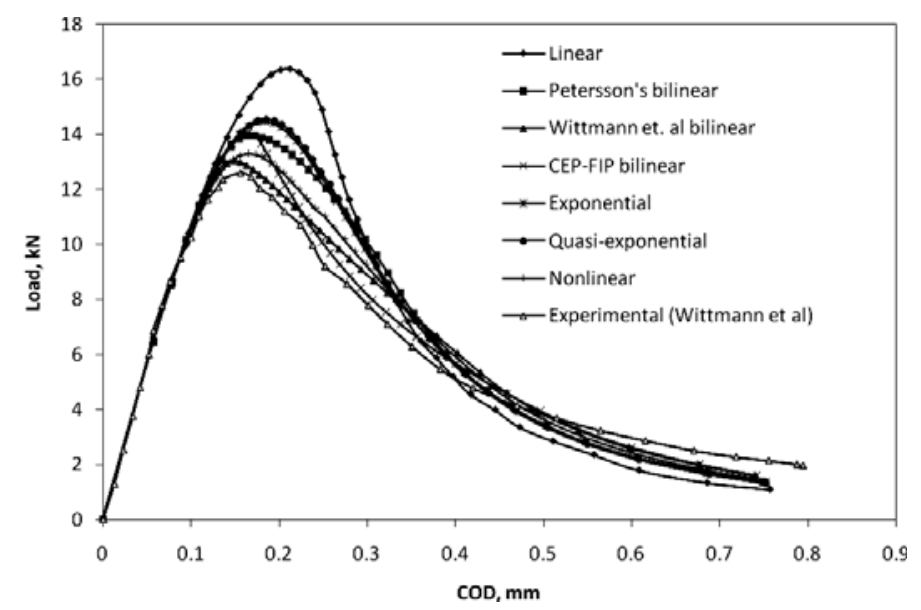

Figure 21. Comparison of P-COD curves obtained from experimental results (Wittmann et al 1988) and the numerical simulation using various softening relations for CT specimen having $D=600 \mathrm{~mm}$ and $a_{o} / D=0 \cdot 5$.

the experiment for the reference specimen. The P-COD curves obtained from the numerical results and the test results are shown in figure 21. It is observed that the ratios of peak load obtained from the numerical results to experimentally observed value are 1.301, 1.099, 1.036, 1.117, 1.148, 1.155 and 1.055 for linear, Petersson (1981) bilinear, Wittmann et al (1988) bilinear, CEB-FIP Model Code (1990) bilinear, exponential (Karihaloo 1995), quasiexponential (Planas \& Elices 1990) and nonlinear (Reinhardt et al 1986) softening functions, respectively. It can be seen that except linear softening, the predicted values of the peak loads are overestimated by approximately $16 \%$. The predicted peak loads are very close (within the accuracy level of 6\%) to the experimental result for the Wittmann et al (1988) bilinear and nonlinear (Reinhardt et al 1986) softening functions. Further, Petersson (1981) bilinear, CEB-FIP Model Code (1990) bilinear, exponential (Karihaloo 1995) and quasiexponential (Planas \& Elices 1990) softening functions yield the peak loads within an accuracy range between 10 and $16 \%$. The predicted value of the peak load using linear softening is overestimated by nearly $30 \%$. It is further observed that the predicted post-peak response are scattered in a relatively narrow band and compare well with the experimentally observed response. However, the terminal potion of experimentally observed softening branch tends to deviate from the numerically predicted values even with all the softening functions used in the numerical modelling.

\section{Conclusion}

The global response and localized fracture behaviour for concrete compact tension specimen of different sizes using cohesive crack model were studied in this paper. Since, the fracture behaviour of concrete varies with the type of softening function used in the cohesive crack model; seven numbers of widely used softening functions were considered in the study. From the present investigation, the following observations could be noticed.

- Using different softening functions (except linear one) it was observed from cohesive crack model that the differences between the highest and lowest peak loads were less 
than about $9 \%$ for concrete compact tension specimen up to $600 \mathrm{~mm}$ size. On an average, the peak load predicted using linear softening was approximately $16 \%$ larger than those obtained using other softening functions (bilinear curves, exponential, quasi-exponential and nonlinear curves).

- Exponential and quasi-exponential softening relations yielded almost the same fracture behaviour.

- The deviation of peak load capacity of normal size concrete specimen using classical LEFM principle from those obtained using cohesive crack model was relatively less for linear softening than those obtained using other softening functions (bilinear curves, exponential, quasi-exponential and nonlinear curves).

- For a given specimen size the fully developed process zone using linear softening was significantly smaller than those obtained using other softening functions (bilinear curves, exponential, quasi-exponential and nonlinear curves). The linear softening yielded the fracture process zone about $30 \%$ and $50 \%$ lower than the bilinear curves, exponential, quasi-exponential and nonlinear softening functions for the size range $200 \mathrm{~mm}$ and $600 \mathrm{~mm}$ respectively.

- The linear softening predicted relatively higher value of crack-tip opening displacement and lower value of the tip stress transfer ratio at peak load than those obtained using the bilinear curves, exponential, quasi-exponential and nonlinear softening functions.

- The peak load gained from the test result was predicted by the cohesive crack model within about $6 \%$ of accuracy using Wittmann et al (1988) bilinear and nonlinear (Reinhardt et al 1986) softening functions whereas those predictions varied in an accuracy range of 10-16\% using Petersson (1981) bilinear, CEB-FIP Model Code (1990) bilinear, exponential (Karihaloo 1995) and quasi-exponential (Planas \& Elices 1990) softening functions. The linear softening curve overestimated the experimentally observed peak load by about $30 \%$.

- The numerically gained softening branch of load-displacement curves using different softening functions agreed well with the experimental observation.

\section{References}

Alfaiate J, Pires E B, Martins J A C 1997 A finite element analysis of non-prescribed crack propagation in concrete. Computers Struct. 63(1): 17-26

ASTM International Standard E399-06 2006 Standard test method for linear-elastic method planestrain fracture toughness $K_{I C}$ of metallic materials. 1-32

Barenblatt G I 1959 The formation of equilibrium cracks during brittle fracture: general ideas and hypotheses, axially symmetric cracks. J. Appl. Math. Mech. 23: 622-636

Bažant Z P 2002 Concrete fracture models: testing and practice. Eng. Fract. Mech. 69: 165-205

Bažant Z P, Kim J-K, Pfeiffer P A 1986 Determination of fracture properties from size effect tests. J. Struc. Eng., ASCE 112: 289-307

Bažant Z P, Oh B H 1983 Crack band theory for fracture of concrete. Mater. Struct. 16(93): 155-177

Bocca P, Carpinteri A, Valente S 1991 Mixed mode fracture of concrete. Int. J. Solids Struct. 27(9): 1139-1153

Brühwiler E, Wittmann F H 1990 The wedge splitting test: a method of performing stable fracture mechanics tests. Eng. Fract. Mech. 35: 117-125

Carpinteri A 1989 Decrease of apparent tensile and bending strength with specimen size: two different explanations based on fracture mechanics. Int. J. Solids Struct. 25: 407-429

Carpinteri A, Colombo G 1989 Numerical analysis of catastrophic softening behaviour (snap-back instability). Comp. Struct. 31(4): 607-636 
CEB-Comite Euro-International du Beton-EB-FIP Model Code 1990, Bulletin D'Information No. 2123/214, Lausanne

Dugdale D S 1960 Yielding of steel sheets containing slits. J. Mech. Phys. Solids 8: 100-104

Gerstle H, Xie M 1992 FEM modelling of fictitious crack propagation in concrete. J. Eng. Mech., ASCE 118(2): 416-434

Gopalaratnam V S, Shah S P 1985 Softening response of plain concrete in direct tension. J. Am. Concrete Inst. 82: 310-323

Hillerborg A, Modeer M, Petersson P E 1976 Analysis of crack formation and crack growth in concrete by means of fracture mechanics and finite elements. Cement Concrete Res. 6: 773-782

Ingraffea A R, Gerstle W H, Gergely P, Saouma V 1984 Fracture mechanics of bond in reinforced concrete. J. Struct. Eng., ASCE 110(4): 871-890

Issa M A, Hammad A M, Chudnovsky A 1993 Correlation between crack tortuosity and fracture toughness in cementitious material. Int. J. Fracture 69: 97-105

Issa M A, Issa M A, Islam M S, Chudnovsky A 2000a Size effects in concrete fracture: Part I, experimental setup and observations. Int. J. Fracture 102: 1-24

Issa M A, Issa M A, Islam M S, Chudnovsky A 2000b Size effects in concrete fracture-Part II: Analysis of test results, Int. J. Fracture 102: 25-42

Issa M A, Issa M A, Islam M S, Chudnovsky A 2003 Fractal dimension-a measure of fracture roughness and toughness of concrete. Eng. Frac. Mech. 70: 125-137

Jenq Y S, Shah S P 1985 Two parameter fracture model for concrete. J. Eng. Mech., ASCE 111(10): $1227-1241$

Kaplan M F 1961 Crack propagation and the fracture of concrete. J. Am. Concrete Inst. 58(5): 591-610

Karihaloo B L 1995 Fracture mechanics and structural concrete. Concrete Design and Construction Series, (Harlow, Essex, England: Longman Scientific \& Technical)

Kesler C E, Naus D J, Lott J L 1972 Fracture mechanics: its applicability to concrete. Proceedings of the International Conference on Mechanical Behaviour of Materials, The Society of Material Science, Kyoto, Japan 4: 113-124

Li Y N, Liang R Y 1994 Peak load determination in linear fictitious crack model. J. Eng. Mech., ASCE 120(2): 232-249

Nallathambi P, Karihaloo B L 1986 Determination of specimen-size independent fracture toughness of plain concrete. Mag. Concrete Res. 38(135): 67-76

Petersson P E 1981 Crack growth and development of fracture zone in plane concrete and similar materials. Report No. TVBM-1006, Division of Building Materials. Lund Institute of Technology, Lund, Sweden

Planas J, Elices M, 1990 Fracture criteria for concrete: mathematical approximations and experimental validation. Eng. Frac. Mech. 35: 87-94

Planas J, Elices M, 1991 Nonlinear fracture of cohesive materials. Int. J. Fracture 51: 139-157

Prasad M V K V, Krishnamoorthy C S 2002 Computational model for discrete crack growth in plain and reinforced concrete. Computer Meth. Applied Mech. Eng. 191: 2699-2725

Reinhardt H W, Cornelissen H A W, Hordijk D A 1986 Tensile tests and failure analysis of concrete. J. Struct. Eng. ASCE 112(11): 2462-2477

RILEM Draft recommendation (50-FMC) 1985 Determination of the fracture energy of mortar and concrete by means of three-point bend test on notched beams. Mater. Struct. 18: 285-290

Using MATLAB, Version 7, The MathWorks, Inc., Copyright 1984-2004

Walsh P F 1972 fracture of plain concrete. Indian concrete J. 46(11): 469-470, 476

Walsh P F 1976 Crack initiation in plain concrete. Mag. Concrete Res. 28: 37-41

Wittmann F H, Rokugo K, Brühwiler E, Mihashi H, Simopnin P 1988 Fracture energy and strain softening of concrete as determined by compact tension specimens. Mater. Struct. 21: 21-32

Xie M, Gerstle W H 1995 Energy-based cohesive crack propagation modelling. J. Eng. Mech., ASCE 121(12): 1349-1358

Xu S, Reinhardt H W 1999a Determination of double-K criterion for crack propagation in quasi-brittle materials, Part I: Experimental investigation of crack propagation. Int. J. Fracture 98: 111-149 
Xu S, Reinhardt H W 1999b Determination of double-K criterion for crack propagation in quasi-brittle materials, Part II: analytical evaluating and practical measuring methods for three-point bending notched beams. Int J. Fracture 98: 151-177

Xu S, Zhang X 2008 Determination of fracture parameters for crack propagation in concrete using an energy approach. Eng. Fract. Mech. 75: 4292-4308 